Running head: FAVOR GIVING AND SOCIAL STATUS

\title{
Reaching the Limits of Reciprocity in Favor Exchange: The Effects of Generous, Stingy, and Matched Favor Giving on Social Status
}

\author{
Kan OUYANG \\ Shanghai University of Finance and Economics \\ Shanghai, China \\ e-mail: ouyangkan@mail.shufe.edu.cn \\ Erica XU \\ Hong Kong Baptist University \\ Kowloon Tong, Kowloon, Hong Kong \\ e-mail: erica_xu@hkbu.edu.hk \\ Xu HUANG \\ Hong Kong Baptist University \\ Kowloon Tong, Kowloon, Hong Kong \\ e-mail: xu.huang@hkbu.edu.hk \\ Wu LIU \\ The Hong Kong Polytechnic University \\ Hung Hom, Kowloon, Hong Kong \\ e-mail:wu.liu@polyu.edu.hk \\ Yipeng TANG \\ Zhongnan University of Economics and Law \\ Wuhan, China \\ e-mail: edyptang@hotmail.com
}

Author note: The first two authors contributed equally to this work, and the order of authorship was determined alphabetically. We thank for Michael Bond and Zhen Zhang for their valuable feedback. Portions of these data have been presented at the 2014 meeting of the Society for Industrial Organizational Psychology. Correspondence concerning this article should be addressed to Erica Xu (erica_xu@hkbu.edu.hk). 


\title{
Reaching the Limits of Reciprocity in Favor Exchange: The Effects of Generous, Stingy, and Matched Favor Giving on Social Status
}

\begin{abstract}
Group members gain social status via giving favors to others, but why and when they do so remain unclear in the literature. Building on social exchange theory and social status literature, we identify three types of favor giving among group members (generous, stingy, and matched) and propose that an affective mechanism (i.e., gratitude) and a cognitive mechanism (i.e., perceived competence) underlie the relationship between favor giving and status attainment. Specifically, generous/stingy favor giving has a linear relationship with status attainment through both gratitude and perceived competence, whereas matched favor giving has a curvilinear relationship with status attainment only through perceived competence. An experimental study and a field study lend support to our propositions. Our study complements the literature by offering a complete picture of how three types of favor giving among group members shape their social status in different ways.
\end{abstract}

Keywords: generous/stingy favor giving, matched favor giving, gratitude, perceived competence, social status 
Social hierarchy is an inevitable phenomenon of group life, and most group members desire to achieve high social status in their groups (Anderson, Hildreth, \& Howland, 2015; Magee \& Galinsky, 2008). Defined as prominence, respect, and deference from other group members (Anderson, John, Keltner, \& Kring, 2001; Magee \& Galinsky, 2008), social status often brings individuals self-esteem, exchange opportunities, promising careers, and even good health (Hardy \& Van Vugt, 2006; Keltner, Gruenfeld, \& Anderson, 2003; Magee \& Galinsky, 2008). It is thus interesting and important to examine what helps group members achieve social status. Existing research has suggested that one important way by which group members can do so is through giving favors, such as offering technical support, advice, information, expertise, and assistance, to coworkers (e.g., Flynn, 2003; Flynn, Reagans, Amanatullah, \& Ames, 2006). Specifically, if individuals give more favors than what they receive, termed as generous favor giving (Flynn, 2003), coworkers are likely to confer these individuals with high status; by contrast, if individuals receive more than they give, coined as stingy favor giving, they are likely to get lower social status (Flynn, 2003; Flynn et al., 2006).

In its explorations of the association between favor giving and status attainment, however, existing research has paid little attention to a third form of favor giving - matched favor giving, defined as a situation in which the favors that one gives out are more or less equal to the favors received from others. This lack of attention is surprising, because social exchange theory suggests that people have a general tendency to strike a balance between what they take and what they give. When individuals perceive that they have over-benefited, they are motivated to restore equity (Gouldner, 1960). Moreover, recent literature in management and psychology has suggested that matched favor giving is a major type of interpersonal exchange, observed even more frequently than generous/stingy favor giving (Cain, Dana, \& Newman, 2014; Grant, 
2013). Cain and colleagues (2014) have estimated that roughly $50 \%$ of generous giving in previous research is not strictly generous, but could be, for example, the manifestation of an obligation to reciprocate a received favor. Such theoretical discussions and empirical evidence have implied that to gain a full picture of favor giving and status attainment, besides generous and stingy favor giving, it is important to consider matched favor giving.

Incorporating different types of favor giving not only offers a complete picture of status attainment among group members, but also provides a unique opportunity to identify the underlying mechanisms linking favor giving and status attainment. On the one hand, focusing only on generous and stingy favor giving, some scholars have used social exchange theory to explain this link with the view that social status is the exchanged resource for favors received yet not reciprocated (Blau, 1963; Flynn, 2003). This view implicitly assumes that gratitude would likely be the driving force behind the association between giving favors and status attainment. Gratitude is "a positive emotional response that is elicited after receiving a recognized benefit" (Spence, Brown, Keeping, \& Lian, 2014, p. 708). Favor receivers tend to feel grateful and thus confer status to givers in exchange for unpaid favors. The validity of this implicit assumption, however, remains unclear (Flynn, 2003), and it is ambiguous whether gratitude could explain the effect of matched favor giving on status attainment. Different from the dynamics in generous/stingy favor giving, in matched favor giving, there are no unpaid favors between exchange parties, and thus gratitude may not be a necessary mechanism leading to status. Therefore, it is critical to examine whether gratitude is a mediating mechanism for each of the various types of favor giving.

On the other hand, in the status literature, researchers have broadly suggested that perceived competence is an important route for status attainment (e.g., Anderson \& Kilduff, 
2009a; Bai, 2016; Cheng, Tracy, Foulsham, Kingstone, \& Henrich, 2013; Ridgeway \& Berger, 1986). People tend to confer status upon members who demonstrate task-related skills and expertise, with the expectation that these competent members will make continuous contributions to the group. The supporting evidence has come mainly from studies on competence traits (e.g., intelligence) and such characteristics as gender, age, and race (Berger, Cohen, \& Zelditch, 1972), although recent discussions have extended to consider behaviors and strategies that make members appear competent (e.g., Anderson \& Kilduff, 2009b; Kalma, Visser, \& Peeters, 1993). The existing literature has remained silent, however, on whether perceived competence helps explain the relationship between favor giving and status attainment, not to mention whether it plays a mediating role equally across different types of favor giving.

In short, there has been little rigorous and systematic investigation on why favor giving influences status attainment among team members. Although previous favor-giving literature (e.g., Flynn, 2003) and social-status research (e.g., Anderson \& Kilduff, 2009a) have suggested two different mediating mechanisms (gratitude and perceived competence), we know little about whether the link between favor giving and status attainment is driven by the gratitude mechanism, or the perceived competence mechanism, or both. It is important to address this research question, because it would help explain group members' affective and cognitive responses along the process of favor giving and status attainment (Flynn, 2003). In addition, favor giving between group members not only entails the social exchange of resources, but also signals individual competence beneficial to the whole group. By examining the effect of favor giving on status attainment, we can potentially integrate the sporadic discussions in the socialexchange and social-status literature to provide a comprehensive answer to the questions of how and why members can gain status in groups through doing favors for colleagues. 
In the present paper, we build a dual-process model (gratitude-competence) to explain the relationship between favor giving and status attainment. More importantly, we propose a linear effect of generous/stingy favor giving on status attainment through both gratitude and perceived competence mechanisms, but a curvilinear effect of matched favor giving on status attainment only through the perceived competence process (see Figure 1). Theorizing and testing such a dual-process model while considering three types of favor giving (generous, stingy, and matched), we contribute to the existing favor-exchange and status-attainment literature in several important ways. First, we develop a more complete picture of different types of favor giving among employees than what has existed in previous literature, particularly in terms of how matched favor giving influences an individual actor's status attainment. We bring this pervasive (Cain et al., 2014; Grant, 2013) yet under-examined condition of favor giving to the forefront of the favor-exchange literature.

Second, we deepen the understanding of why and how favor giving contributes to status attainment in work groups by proposing and testing the dual-process model. By integrating social exchange theory and status research, the dual-process model presents two routes that can differentially link different types of favor giving with social status. On the one hand, although prior research has demonstrated the effect of generous/stingy favor giving on status attainment (e.g., Flynn, 2003), our dual-process model can explain why this is so. On the other hand, differentiating affective and cognitive mechanisms helps explain how matched favor giving, the under-studied type of favor giving, affects social status in a curvilinear fashion.

Finally, our paper also contributes to the literature on helping behavior and organizational citizenship behavior (OCB). Favor giving is conceptually equivalent to helping behavior and OCB towards individuals. Past research on helping behavior and OCB has focused primarily on 
the consequences from the employers' perspective, in such forms as performance evaluations, organizational rewards, and career promotion (e.g., Allen \& Rush, 1998; Podsakoff, MacKenzie, \& Hui, 1993; Seibert, Kraimer \& Crant, 2001). We complement this literature by examining the consequences from peers' perspective and demonstrating that how employees' helping behavior or OCB affects their social standing in groups.

\section{Theory and Hypotheses}

\section{A Dual-Process Model of Favor Giving and Social Status}

We conceptualize favor giving as influencing status attainment through gratitude, which is a prototypical affective reaction to having received favors (Fisher, Nadler, \& Whitcher-Alagna, 1982; Lawler, 2001; Lawler \& Thye, 1999). In the social-exchange literature, gratitude is conceptualized as an emotional outcome in response to others' prosocial actions on one's behalf that subsequently motivates people to act in a prosocial manner (Lawler \& Thye, 1999;

McCullough, Kilpatrick, Emmons, \& Larson, 2001; Spence et al., 2014). Simmel (1950) and Gouldner (1960), for example, regarded gratitude as an emotional resource for people to maintain reciprocity obligations. People are socialized to experience gratitude toward people who bring them benefits and to remember if the reciprocation has not yet been met (McCullough et al., 2001). Thus, favor giving induces receivers' gratitude toward givers, builds interpersonal affiliations, and enhances affinity (Lawler, Thye, \& Yoon, 2000; Molm \& Cook, 1995).

Triggered by gratitude, favor receivers tend to reciprocate unpaid favors with various exchangeable resources, such as prominence, respect, and deference. According to Foa and Foa's $(1974,1980)$ typology of social resources, favors and social status are both social resources that can be exchanged. When favor receivers cannot directly reciprocate favors, they can confer status to the givers to convey gratitude, as a vital means to fulfill the reciprocity expectation 
(Blau, 1963); meanwhile, it is also an important way to maintain and improve the relationship with generous givers, with the expectation that givers will continue advancing receivers' interests (Algoe \& Haidt, 2009; Algoe, Haidt, \& Gable, 2008).

In addition to gratitude, perceived competence is another key route to gain social status within groups (see the review by Bai, 2016). Status researchers have long suggested that demonstrating superior task-relevant skills and expertise induces positive expectations that the competent group member is instrumental and valuable in accomplishing group tasks and goals, and thus group members are likely to award the member with status (e.g., Wojciszke, Abele, \& Baryla, 2009). Individuals with high intelligence, for example, are likely to attain high social status. Recent research has also suggested that people gain higher status by engaging in behaviors and strategies that make them appear more competent, such as making more suggestions, speaking in more assertive tones, and making more direct eye contact (e.g., Anderson \& Kilduff, 2009b; Kalma et al., 1993). We posit that favor giving may influence status attainment through perceived competence. Through favor giving, actors demonstrate their capability, skills, and expertise in solving work-related problems, which shapes others' evaluations and judgments of actors' ability, reliability, and predictability in future transactions (e.g., Schaubroeck, Lam, \& Peng, 2011). Thus, group members are likely to defer to and confer status on favor givers.

In summary, the proposed dual-process model elaborates perceived competence and gratitude as two mechanisms linking favor giving and status attainment. In addition, we further argue that how the two mechanisms work is contingent upon the type of favor giving.

\section{Generous/Stingy Favor Giving and Social Status}

Normally, group members can quickly spot who are the givers and who are the takers. 
Those who give more favors to others than what they receive from others are "givers," and those who do the opposite are "takers" (Grant, 2013). Research has consistently shown that "givers" attain higher status than "takers" (e.g., Blau, 1963; Flynn, 2003). We argue that both gratitude and competence mechanisms explain status attainment in generous/stingy favor-giving.

Basing their arguments on social exchange theory, researchers have argued that individuals tend to offer status to givers in exchange for received yet not returned favors (e.g., Blau, 1963, 1964; Flynn, 2003). Although individuals tend to exchange the same resource type (i.e., favors in exchange for favors), in generous/stingy favor giving, favor receivers are unable to reciprocate the same amount of favors received from others. Favor receivers usually feel gratitude toward givers when there remain unpaid favors, and this feeling functions as an automatic reminder that receivers need to fulfill their role in providing reciprocity in social exchanges (Gouldner, 1960; Simmel, 1950).

Motivated by gratitude, favor receivers tend to reciprocate generous favor givers by conferring givers with social status, which is a resource that is both available to receivers and typically perceived as valuable in peer relationships at the workplace (e.g., Flynn, 2003; Hardy \& Van Vugt, 2006). Furthermore, recent theoretical development on gratitude has argued that gratitude has a social function in "reminding of a known good relationship partner, and helps to bind recipient and benefactor closer together" (Algoe, 2012, p. 457). Gratitude helps favor receivers solidify and improve their connections with generous givers, whom they believe will continue providing them with help and support (Algoe, 2012; Algoe \& Haidt, 2009; Algoe et al., 2008). Thus, in generous favor giving, gratitude may lead receivers to show deference, respect, and submission to generous givers in "return" for the favors received yet not reciprocated or the favors that receivers are unable to repay (e.g., Algoe \& Haidt, 2009; Baumgarten-Tramer, 1938). 
Moreover, social-status literature has suggested that perceived generous favor giving may elicit pleasant feelings in favor receivers, which intuitively make them willing to express deference and submission to generous givers, with the expectation that generous givers will continue advancing their interests (e.g., Bai, 2016).

Our above discussions suggest that in generous favor giving, receivers feel gratitude toward givers and thus confer high status on them. By contrast, stingy favor giving tends not to induce group members' feelings of gratitude, thereby inhibiting the acts of status conferral from others (e.g., Flynn, 2003; Lawler et al., 2000). We therefore propose that gratitude mediates the relationship between generous/stingy favor giving and social status.

We further propose that generous/stingy favor giving may influence givers' social status through perceived competence. Favor givers help others solve work-related problems, fulfill responsibilities that others cannot, share knowledge and expertise with peers, and even show competitive energy and vigor at work by doing more (Flynn, 2003; Hardy \& Van Vugt, 2006; Smith \& Bird, 2000). Generous favor giving thus demonstrates givers' competence that is salient and important for task-related interactions, which shapes interacting parties' evaluations and judgments of givers' value in the exchange relationship and generates positive expectations of their dependability and ability in future interactions. As a result, interacting parties are likely to confer status to generous givers as reciprocation (Anderson \& Kilduff, 2009a; Flynn, 2003; Flynn et al., 2006). Conversely, stingy favor givers may signal their lack of task-related competence, ability, and sense of responsibility by taking others' favors while offering limited contributions in return, resulting in lower social status. Taking both gratitude and perceived competence perspectives, we propose that:

Hypothesis 1a: Social status is higher when one gives more than receives (i.e., generous 
favor giving) than when one receives more than gives (i.e., stingy favor giving).

Hypothesis $1 b$ : Gratitude mediates the relationship between generous/stingy favor giving and favor givers' social status.

Hypothesis 1c: Perceived competence mediates the relationship between generous/stingy favor giving and favor givers' social status.

\section{Matched Favor Giving and Social Status}

In matched favor giving, favors that an individual gives to others are tantamount to those received from others. Overall, an individual has no "unpaid" favors in the team, and thus previous arguments based on the gratitude mechanism by which receivers reciprocate unpaid favors with social status cannot explain how matched favor giving affects social status. We argue that even though others do not owe any favors to this actor, the act of favor giving itself still advertises the actor's competence to others, and thus others are likely to generate positive expectations of the actor's value in future interactions. They may confer social status on the actor, on the one hand, to develop a long-term, high-quality relationship to ensure future contributions from this competent member, and, on the other hand, to acknowledge and respect the actor's competence and contributions (Anderson \& Kilduff, 2009a; Bunderson, 2003; Ridgeway, 2001). We therefore propose that perceived competence, rather than gratitude, underlies the relationship between matched favor giving and social status. Although we generally expect matched favor giving and status attainment to have an overall positive association, we predict that the positive relationship will taper off at a high frequency of matched favor giving.

The competence-based view of peer favor exchange posits that people assess and evaluate an actor's key characteristics that are important for task- or performance-related interactions. This evaluation process is likely to be influenced, however, by the extent to which 
others possess similar characteristics. Social evaluation theory contends that perceived scarcity leads to more extreme evaluative judgments (Brannon \& Brock, 2001; Ditto \& Jemmott, 1989; Pettigrew, 1967). When many others exhibit a particular behavior frequently, its distinctiveness becomes less salient. Achieving a grade of "A+," for example, is less likely to be perceived as an accomplishment when one half rather than one tenth of the class members have attained it, and thus the grade becomes a less salient indicator of performance competence.

Likewise, when matched favor giving reaches a higher frequency level, the focal actor's reflected competence and ability tend to diminish for two reasons. First, a higher frequency level of matched favor giving suggests that other coworkers are also capable of continuously and increasingly offering favors. Thus the competence signal of the focal giver's favor giving becomes less salient. A higher frequency level of matched favor giving demonstrates the overall competence of everyone in the group, thus diluting the focal actor's competence. Therefore, the focal actor's favor giving is less likely to proportionately translate into increasingly strong competence cues and elicit status conferral. Second, as matched favor giving reaches a high frequency level, everyone is likely to focus more on his/her own contributions, while underestimating and devaluing other members' contributions (Ross \& Sicoly, 1979). As a result, a further increase in matched favor giving will not lead team members to see their exchange partners as being ever-increasingly competent, and vice versa. Thus, overall, at a higher frequency level of matched favor giving, individuals' perceived competence is unlikely to increase in accordance with their increased favor giving, and thus individuals are unlikely to obtain increasing social status at this point. Taken together, our reasoning, based on existing theory and past research, suggests that the positive relationship between matched favor giving and status tends to taper off after the frequency of matched favor giving achieves a high level and 
that perceived competence mediates the curvilinear link between matched giving and status. We thus predict that:

Hypothesis 2a: There is a curvilinear relationship between matched favor giving and favor givers' social status, such that the overall positive relationship is attenuated at higher levels of matched favor giving.

Hypothesis 2b: Perceived competence mediates the curvilinear relationship between matched favor giving and favor givers' social status.

We conducted two studies to test our hypotheses. Study 1 is an experimental study, in which we manipulate the types of favor giving (i.e., generous/stingy favor giving and matched favor giving) to assess their respective impacts on the mediators (i.e., gratitude and perceived competence) and the dependent variable (i.e., social status). Study 2 is a field study with multisource, time-lagged survey data to increase the external validity of our findings.

\section{Study 1 Method}

\section{Sample and Procedure ${ }^{1}$}

We recruited participants from Prolific, a crowdsourcing community for survey and research (https://prolific.ac/). Using Prolific's prescreening tool, we restricted participation to people who were working full time in non-managerial positions. A total of 271 participants from the United States, England, and Australia were initially invited to participate in our study in exchange for US\$1. This study combined the critical incident technique (Flanagan, 1954) with random assignment to experimental conditions. This technique requires participants to recall events or experiences with certain characteristics and describe them in detail. Participants then respond to survey questions. This technique has been widely used in experimental studies (e.g.,

\footnotetext{
${ }^{1}$ Studies 1 and 2 both received ethical approval from the university of the third author to conduct the surveys.
} 
Casciaro, Gino, \& Kouchaki, 2014; Liang, Lian, Brown, Ferris, Hanig, Keeping, 2016; Mayer, Greenbaum, Kuenzi, \& Shteynberg, 2009; Wellman, Mayer, Ong, \& DeRue, 2016).

We manipulated the types of favor giving by providing participants with specific instructions about the type of favor giving to recall (see Appendix). To test the curvilinear relationship between favor giving and social status, we manipulated three conditions for matched favor giving: low, medium, and high levels of matched favor giving. To be consistent, we manipulated three conditions for generous/stingy favor giving, including the "least generous" condition, indicating actors received much more than they gave (i.e., stingy favor giving); the "medium generous" condition, and the "high generous" condition (i.e., generous favor giving).

Participants were randomly assigned to one of the six conditions: They were informed that their task concerned visualizing one of their coworkers who fits the description of the assigned condition. If they could recall such a colleague, they were asked to "write several sentences to describe how you and this colleague helped each other at work." After completing the recall task, respondents rated perceived competence, gratitude toward this colleague, and this colleague's social status and reported their age, gender, and relationship length with this colleague (see Appendix for the detailed flow of the experiment procedure). If they could not recall such a described colleague, they were excluded from the study; we excluded 15 participants for this reason. Moreover, following Meade and Craig's (2012) recommendation, we also excluded six participants who responded carelessly (i.e., those who failed the direct response measure [e.g., please respond with "very frequently"]). The final sample consisted of 250 participants $(49 \%$ female) who were assigned to six conditions: least generous $(\mathrm{N}=50)$, medium generous $(\mathrm{N}=37)$, high generous $(\mathrm{N}=38)$, low matched favor giving $(\mathrm{N}=44)$, medium matched $(\mathrm{N}=39)$, or high matched $(\mathrm{N}=42)$. Participants' mean age was 34.37 years $(S D=$ 
9.55), and the mean length of the dyadic relationship between participants and their coworkers with whom they exchanged favors was 3.83 years $(S D=3.73)$.

Favor-giving manipulation. We restricted the favor giving interactions to those that had occurred at work within the last three months. Three examples of favor giving were provided for each condition (see Appendix; Lee \& Allen, 2002; Podsakoff, Ahearne, \& MacKenzie, 1997). For example, in the condition of high generous favor giving, the participant was asked to visualize a colleague who had frequently helped the participant, but the participant rarely offered this colleague the kinds of help that had been given (see Appendix for the full instructions).

\section{Measures}

Perceived competence. We used a three-item, five-point scale adapted from Stone and Stone (1985) to measure perceived competence. The scale contains three bi-polar items to describe performance-related capabilities, such as "not efficient at all versus very efficient at one's job" $(\alpha=.91)$.

Gratitude. We used a three-item, five-point scale adapted from Cheng and colleagues (2004) to measure gratitude. A sample item is: "I feel grateful to him/her" (1 = strongly disagree, $5=$ strongly agree; $\alpha=.82$ ).

Social status. A four-item, seven-point scale was used to measure status (Flynn, 2003). A sample item is "How much influence does this person exert over decisions at work?" $(1=$ does not affect decisions; $7=$ has a great deal of influence; $\alpha=.85)$.

\section{Study 1 Results}

\section{Manipulation Checks}

Regarding generous/stingy favor-giving manipulations, participants in the high generous favor-giving condition $(M=4.03, S D=.64)$ were significantly more inclined than those in the 
medium generous condition, $M=3.19, S D=.70, t(73)=5.42, p<.001, d=.84$, or those in the least generous condition, $M=1.84, S D=1.06, t(86)=11.29, p<.001, d=2.19$, to report the colleague as being generous in favor giving (e.g., "How frequently did this colleague help you more than you help him/her?" [1 = almost never; 7 = very often $])$. Also, the recalled colleague in the medium generous condition was assessed as being significantly more generous than the one in the least generous condition, $t(85)=6.74, p<.001, d=1.35$.

Regarding matched favor-giving manipulations, participants in the high matched favorgiving condition $(M=4.50, S D=.51)$ were significantly more inclined than those in the medium, $M=4.23, S D=.67, t(79)=2.06, p<.05, d=.27$, and low, $M=3.59, S D=.84, t(84)=$ $6.02, p<.001, d=.91$, matched conditions to report a higher frequency level of equally exchanged favors with the recalled colleague (e.g., "How frequently did you and this colleague equally help each other?" [1 = almost never; 7 = very frequently and equally $])$. Also, participants

in the medium matched condition were significantly more inclined than those in the low matched condition, $t(81)=3.80, p<.01, d=.64$, to report a higher frequency level of equally exchanged favors with the colleague recalled. Thus, our manipulations of favor giving were successful.

\section{Hypothesis Testing}

To test Hypothesis 1a, we conducted independent-samples t-tests to examine the differences of status in the three generous/stingy favor-giving conditions. The results show that social status was significantly higher in the high generous favor-giving condition $(M=4.68, S D$ $=.35)$ than in the medium generous, $M=4.10, S D=.48, t(73)=6.09, p<.001, d=.58$, and the least generous, $M=3.09, S D=1.16, t(86)=8.82, p<.001, d=1.59$, conditions and that status was significantly higher in the medium generous condition than in the least generous condition, $t$ $(85)=4.97, p<.001, d=1.01$ (see Table 1). We also applied hierarchical regression analyses to 
confirm the results, and found that generous/stingy favor giving was positively related to social status $(\beta=.58, p<.001)$. Thus, Hypothesis 1a is supported.

Similarly, as shown in Table 1, the patterns of gratitude and perceived competence for the three generous/stingy favor-giving conditions were similar to those for social status (also see Figure 2), which suggests that generosity leads to higher levels of gratitude and perceived competence. To test the mediating effects of gratitude and perceived competence, regression analyses showed that the coefficient of generous/stingy favor giving on social status dropped from $.58(p<.001)$ to $.15(p<.01)$ after entering the two mediators. We then employed a bootstrapping approach to further confirm the indirect effects of gratitude and perceived competence (Preacher \& Hayes, 2008). A bootstrapping based on 5,000 resamples demonstrated that the indirect effects of generous/stingy favor giving on status via gratitude, indirect effect $=.13, p<.01,95 \% \mathrm{CI}=[.05, .24]$, and perceived competence, indirect effect $=.30, p<.001$, $95 \% \mathrm{CI}=[.19, .44]$, were significant. Hypotheses $1 \mathrm{~b}$ and $1 \mathrm{c}$ are therefore supported.

Hypothesis 2 a proposes a decreasingly positive curvilinear relationship between matched favor giving and social status. The $t$-test results show that social status was significantly lower in the low matched favor-giving condition $(M=4.19, S D=.71)$ than in the medium, $M=4.48, S D$ $=.47, t(81)=2.18, p<.05, d=.29$, and high, $M=4.52, S D=.45, t(84)=2.57, p<.05, d=.33$, matched favor-giving conditions, but status was not significantly different between the medium and high matched conditions, $t(79)=.37, n . s ., d=.04$ (see Table 1). Hierarchical regression analyses further confirmed that matched favor giving $(\beta=.19, p<.001)$ and its squared term $(\beta$ $=-.10, p<.05$ ) were both significantly related to social status. Simple slope tests (Aiken \& West, 1991) showed that the regression line was positive and significant $(\beta=.32, p<.001)$ at a low level of matched favor giving (1 SD below the mean), whereas the simple slope was not 
significantly different from zero $(\beta=-.03$, n.s. $)$ at a high level of matched favor giving (1 SD above the mean). The results support Hypothesis 2a.

Next, we tested the mediating effect of perceived competence in the relationship between matched favor giving and social status. As shown in Table 1, the pattern of perceived competence in the three conditions of matched favor-giving was similar to that of social status, suggesting a curvilinear relationship (see Figure 3). We also applied regression analyses to further confirm the results and found that matched favor giving had a curvilinear relationship with competence $\left(\beta_{\text {favor-giving }}=.15, p<.01 ; \beta_{\text {favor-giving }^{2}}=-.09, p<.05\right)$. The simple slope tests (Aiken $\&$ West, $1991)$ confirmed a positive diminishing effect $\left(\beta_{\text {low }}=.38, p<.001 ; \beta_{\text {high }}=-.00\right.$, n.s. $)$. A regression test also showed that when entering perceived competence as the mediator, perceived competence was significantly related to status $\left(\beta=.28, p<.01, \Delta \mathrm{R}^{2}=.15\right)$, but the squared term of matched favor giving became insignificant. A bootstrapping procedure based on a 5,000 resamples further demonstrated the indirect effect of perceived competence (Hayes \& Preacher, 2010), indirect effect $=.07, p<.05,95 \% \mathrm{CI}=[.02, .14]$. Hence, perceived competence mediated the curvilinear relationship between matched favor giving and social status, supporting Hypothesis $2 b$. Regarding gratitude, as shown in Table 1, the value of gratitude showed no differences between any two conditions, and therefore, gratitude was not the mediator in the relationship between matched favor giving and status.

\section{Study 2 Method}

\section{Sample and Procedure}

We collected data from two samples: employees of a large, state-owned telecommunications company in central China and teachers of two kindergartens in southern China. We adopted a round-robin design (Warner, Kenny, \& Stoto, 1979) to avoid common 
method bias. We focused on favor giving among group members, and thus we excluded group leaders from the round-robin data collection. In a group with four members, for example, member A reported how often members B, C, and D gave favors to him/her; member B reported how often members A, C, and D gave favors to him/her; and so on. The procedure allowed us to calculate A's favor giving by averaging B's, C's, and D's ratings of received favors from A, and to calculate A's favor receiving by averaging A's ratings of received favors from B, C, and D. To be more specific, in the polynomial regression equation $\left(Z=b_{0}+b_{1} X+b_{2} Y+b_{3} X^{2}+b_{4} X Y+\right.$ $\left.\mathrm{b}_{5} \mathrm{Y}^{2}+e\right), \mathrm{X}$ indicates an individual member's favor receiving from all other members, selfreported by the focal member. Y indicates this individual member's favor giving to all other members, which was other-rated by all the other group members, because all other members reported how many favors they had received from this focal member; in other words, $\mathrm{Y}$ indicates this focal member's favor giving to all other members. $\mathrm{Z}$ indicates this focal member's social status, which was other-rated by all other members at Time 2 .

We collected data with a three-month time lag. At Time 1, employees rated favor giving, gratitude, and perceived competence, as well as data on control variables. At Time 2, employees rated social status for their group members. We met personally with respondents to brief them about our purposes and the survey procedures. Respondents received a cover letter explaining the study, a questionnaire, and a return envelope. To ensure confidentiality, respondents sealed the completed questionnaires in envelopes and returned them to us directly on site.

For the telecommunications company sample, we randomly distributed paper-and-pencil surveys to 250 employees from 50 groups across departments with a variety of functions, such as sales, finance, and technology maintenance. The group size ranged from 6 to 10 employees. For the kindergarten sample, we distributed surveys to 183 teachers from 47 work groups, with the 
group size ranging from 3 to 5 people. The final sample consisted of 362 employees from 89 groups $\left(\mathrm{N}_{\text {telecommunications }}=220\right.$ from 49 groups; $\mathrm{N}_{\text {kindergarten }}=142$ from 40 groups $)$, yielding a response rate of $84 \%$. Among participants, $30 \%$ were male; $77 \%$ had a college degree or above; the mean age was 33.59 years $(S D=9.78)$, and the mean organizational tenure was 11.02 years $(S D=7.50)$.

\section{Measures}

All measures used in this research were originally developed in English. Bilingual experts first translated the measures into Chinese and then back-translated them into English. We then compared the back-translated English version with the original English version for equivalence and agreement (Brislin, Lonner, \& Thorndike, 1973).

Favor giving. Using a five-point, four-item scale adapted from Podsakoff et al. (1997) and Lee and Allen (2002), respondents rated the extent to which they had received favors from each member of their group. To avoid the rating fatigue that can occur from a round-robin process, we selected four of the seven items from the original scale of helping behavior that more specifically describe work-related favors. For example, "[X] gives his/her time to help me with work-related problems" $(1=$ never $; 5=$ very often; $\alpha=.94)$. We then averaged how often other group members reported receiving favors from the focal member (i.e., the focal member's favor giving) and how often the focal member reported receiving favors from other group members (i.e., the focal member's favor receiving). Thus we derived a measure of favor giving and a measure of favor receiving for each member.

Perceived competence. We measured it with the same scale used in Study $1(\alpha=.95)$. We aggregated team members' ratings to obtain an averaged score for the focal member. We found sufficient statistical support for the aggregation $\left(\mathrm{ICC} 1=.38 ; \mathrm{ICC} 2=.75 ; \mathrm{r}_{\mathrm{wg}(\mathrm{j})}=.98\right)$. 
Gratitude. We measured gratitude with the same scale used in Study $1(\alpha=.89)$. We aggregated the ratings from members of a focal individual's group to obtain an average gratitude score for the focal individual $\left(\mathrm{ICC} 1=.27 ; \mathrm{ICC} 2=.65 ; \mathrm{r}_{\mathrm{wg}(\mathrm{j})}=.95\right)$.

Social status. Social status was assessed with the same scale as that in Study $1(\alpha=.94)$. We then aggregated the ratings from all group members to obtain an average social status score $\left(\mathrm{ICC} 1=.47 ; \mathrm{ICC} 2=.81 ; \mathrm{r}_{\mathrm{wg}(\mathrm{j})}=.95\right)$.

Control variables. We controlled for age, gender, education, and organizational tenure, because these variables may influence interactions between peers and, in turn, social status (e.g., Ehrhart, Bliese, \& Thomas, 2006; Flynn, 2003). Given that individuals' perceptions of favor giving and receiving may be subject to the level of favors in the group overall, we also controlled for group-level favor receiving, which was obtained by averaging each group member's individual favor receiving. Finally, we controlled for employees' past performance, as employee performance may substantially affect their status in the group (e.g., Berger, Rosenholtz, \& Zelditch, 1980; Bunderson, 2003). In so doing, we tried to minimize the possibility that employees' social status may bias their reported levels of favor receiving. Employees' past performance was rated by their supervisors, using a three-item, seven-point scale (Motowidlo \& Van Scotter, 1994; $\alpha=.94)$. Also, to control for the effect of the organization type, we created a dummy variable.

\section{Data Analyses}

Cross-level polynomial regressions. Polynomial regression is used to examine the congruence effect (i.e., fit, match, similarity, or agreement) between two constructs on a third construct and the incongruence effect (i.e., difference, mismatch, or disagreement) of two constructs on a third construct (e.g., Edwards \& Cable, 2009; Edwards \& Parry, 1993). 
Researchers have often used the polynomial regression method to examine the outcomes of (a) self-observer rating congruence and discrepancies in multisource feedback instruments (e.g., Ostroff, Atwater, \& Feinberg, 2004), (b) person-environment fit and misfit (e.g., Edwards \& Rothbard, 1999; Jansen \& Kristof-Brown, 2005), and (c) supervisor-subordinate congruence and incongruence of their perceptions or personal traits (e.g., Matta, Scott, Koopman, \& Conlon, 2014; Zhang, Wang, \& Shi, 2012). Our study focused on the incongruence effect (i.e., generous/stingy favor giving) and the congruence effect (i.e., matched favor giving) of one's favor giving and favor receiving on social status and the underlying mechanisms.

Given that individual members were nested within groups, we conducted cross-level polynomial regression (Jansen \& Kristof-Brown, 2005; Zhang et al., 2012) and created threedimensional response surface plots (Edwards \& Parry, 1993) to examine and display how generous/stingy favor giving and matched favor giving affect social status. According to this technique, the dependent variables (e.g., social status) were regressed on control variables, as well as five polynomial terms: favor giving (FG), favor receiving (FR), favor giving times favor receiving $(\mathrm{FG} * \mathrm{FR})$, favor giving squared $\left(\mathrm{FG}^{2}\right)$, and favor receiving squared $\left(\mathrm{FR}^{2}\right)$. In addition, to reduce multicollinearity and to facilitate the interpretations of the results, FG and FR were standardized around the grand mean within each sample and the second-order terms were calculated afterwards. After performing polynomial regressions, we examined the slopes and curvatures along two critical lines: the generous/stingy line $(\mathrm{FG}=-\mathrm{FR})$ and the matched line $(\mathrm{FG}$ =FR). Following Edwards and Parry (1993), when the three second-order polynomial terms (i.e., $\mathrm{FG}^{*} \mathrm{FR}, \mathrm{FG}^{2}$, and $\mathrm{FR}^{2}$ ) are jointly significant (i.e., the $F$ score for the three second-order terms is significant), the slope along the generous/stingy line is significantly positive, and the curvature along this line is not significant, we can conclude that group members who give more than they 
receive are conferred higher status than those who receive more than they give (i.e., Hypothesis 1a). Moreover, when the slope along the matched line is significantly positive and the curvature along this line is significantly negative, it suggests a surface with a downward curve along the matched line (i.e., Hypothesis 2a). Finally, we plotted the response surface graphs to visually depict the results of the cross-level polynomial regression.

Mediation test using the block variable approach. To test the mediating effects of gratitude and perceived competence (Hypotheses 1b, 1c, and 2b), we employed the block variable approach (Edwards \& Cable, 2009; Zhang et al., 2012). This block method is particularly useful when, for example in our case, the independent variable of the mediation model involves linear and curvilinear components of more than one variable (congruence and incongruence of favor giving and favor receiving). One solution is to compute a composite variable (i.e., block variable) to represent this group of components and then use this composite variable in the mediating analysis (Edwards \& Cable, 2009; Heise, 1972; Zhang et al., 2012).

Specifically, we obtained a single coefficient to represent the joint effect of the five polynomial terms $\left(\mathrm{FG}, \mathrm{FR}, \mathrm{FG}^{2}, \mathrm{FG} * \mathrm{FR}, \mathrm{FR}^{2}\right)$ by combining the five terms into a block variable. The block variable is a weighted linear composite calculated from the estimated regression coefficients in the polynomial regression. After generating the block variable, we reran the cross-level polynomial regression and obtained the standardized regression coefficient of the block variable as the path coefficient for the mediation analysis. The indirect effect of favor giving (both the generous/stingy type and matched favor giving) on social status via the mediator can be calculated as a product of the coefficient of the block variable on the mediator and the coefficient of the mediator on status when we include the direct effect of favor giving (generous/stingy and matched) in the regression. We tested the significance of the indirect effects 
by using the Monte Carlo method and estimated 95\% confidence intervals for the indirect effects, based on 20,000 simulated samples (Preacher \& Selig, 2012; Selig \& Preacher, 2008).

\section{Study 2 Results}

Table 2 presents means, standard deviations, and correlations among key variables. We first conducted confirmatory factor analyses (CFAs) to examine the distinctiveness of the four employee-rating variables (i.e., favor giving, gratitude, perceived competence, and social status). The hypothesized four-factor model was compared with a series of alternative models, including three three-factor models that had any two variables combined; one two-factor model that had gratitude, perceived competence, and status combined; and one single-factor model. The hypothesized four-factor model yielded a better model fit $\left(\chi^{2}=437.88, d f=60\right.$, CFI $=.98$, TLI $=.97, \mathrm{RMSEA}=.07)$ than all the alternative models, and none the alternative models reached acceptable model fit. We then followed the procedure recommended by Shanock and colleagues (2010) to determine the proportions of the three types of favor giving. We found adequate cases for each type of favor giving to allow us to perform a polynomial regression (Generous $=36.5 \%$; Stingy $=29.3 \%$; Matched $=34.3 \%)$.

Hypothesis 1a predicts the effect of generous/stingy favor giving on social status. Model 3 in Table 3 displays the estimated coefficients, as well as the slopes and curvatures along the generous/stingy line and the matched line for social status, based on which the response surface

is plotted (Figure 4). As shown, the three second-order polynomial terms (i.e., FG * FR, FG ${ }^{2}$, and $\left.\mathrm{FR}^{2}\right)$ were jointly significant $(\mathrm{F}=8.44, p<.05)$; the slope along the generous/stingy line was significantly positive (slope $=.46, p<.001)$ with an insignificant curvature (curvature $=.04$, n.s.), meaning that status increases along the generous/stingy line. The results indicate that group members who were generous in giving favors had higher status than those who received more 
than they gave, thus supporting Hypothesis 1a.

Hypothesis 2a predicts a decreasingly positive curvilinear relationship between matched favor giving and social status. As Model 3 of Table 3 shows, the significant slope (slope $=.44, p$ $<.001$ ) and curvature (curvature $=-.18, p<.01$ ) along the matched line indicate a surface with a downward curve along the matched line. We then conducted simple slope tests (Aiken \& West, 1991) to confirm the direction of the surface along the matched line in Figure 4 . When matched favor giving had a low frequency (1 SD below the mean), the simple slope of the regression curve had a significant and positive value $(\beta=.80, p<.001)$; when the frequency of matched favor giving was high (1 SD above the mean), the simple slope was not significant $(\beta=.16$, n.s. $)$. The results suggest that the positive relationship between matched favor giving and givers' status leveled off at higher levels of matched favor giving. Hence, Hypothesis $2 \mathrm{a}$ is supported.

To test the mediating hypotheses (Hypotheses 1b, 1c, and 2b), we first ran two regressions using the five polynomial terms to predict perceived competence and gratitude, as well as one regression to link the two mediators with social status. The results of Model 1 in Table 3 support a linear relationship between generous/stingy favor giving and perceived competence (the generous/stingy line: slope $=.38, p<.001 ;$ curvature $=-.02$, n.s. $)$ and a curvilinear relationship between matched favor giving and perceived competence (the matched line: slope $=.40, p<.001 ;$ curvature $=-.12, p<.05 ;$ simple slope test: $\beta_{\text {low }}=.63, p<.001 ; \beta_{\text {high }}$ $=.16$, n.s. $)$. The results of Model 2 of Table 3 indicate that gratitude has linear, positive relationships along both the generous/stingy line $($ slope $=.42, p<.001$; curvature $=-.03$, n.s. $)$ and the matched line (slope $=.30, p<.001$; curvature $=-.03$, n.s. $)$. Figures 5 and 6 plot the response surfaces of perceived competence and gratitude.

We then used the block variable approach and the Monte Carlo method to further test the 
mediating effects of gratitude and perceived competence. Specifically, using the estimated coefficients of the five polynomial terms generated in Model 1 in Table 3, we obtained a block variable (i.e., block variable $=.39 * \mathrm{FG}+.01 * \mathrm{FR}-.00 * \mathrm{FG}^{2}-.05 * \mathrm{FG} * \mathrm{FR}-.07 * \mathrm{FR}^{2}$ ). We then regressed perceived competence on the block variable. As such, we obtained a single coefficient that represents the joint effect of generous/stingy/matched favor giving on perceived competence $(\gamma=.50, p<.001)$. We used the same procedure to generate the coefficients of the joint effect on gratitude $(\gamma=.49, p<.001)$ and social status $(\gamma=.25, p<.001)$. These coefficients were used to calculate the indirect effects of generous/stingy/matched favor giving on social status via the two mediators in the Monte Carlo tests.

As shown in Table 4, the indirect effect of generous/stingy/matched favor giving on status via perceived competence was significant (indirect effect $=.22, p<.001,95 \% \mathrm{CI}=[.15, .30]$ ). The patterns of the effects of generous/stingy/matched favor giving on perceived competence were similar to those of the effects of generous/stingy/matched favor giving on status (i.e., a linear relationship along the generous/stingy line and a curvilinear relationship along the matched line). Hence, perceived competence not only explained the linear effect of generous/stingy favor giving on status, but also accounted for the curvilinear link between matched favor giving and status, supporting Hypotheses $1 \mathrm{~b}$ and $2 \mathrm{~b}$. Regarding gratitude, Table 4 shows that the indirect effect of generous/stingy/matched favor giving on status via gratitude was also significant (indirect effect $=.06, p<.01,95 \% \mathrm{CI}=[.03, .10]$ ). Since the earlier test showed that the relationship between matched favor giving and gratitude did not present a curvilinear relationship, we conclude that gratitude mediated only the relationship between generous/stingy favor giving and social status, supporting Hypothesis 1c.

\section{Discussion}


In this research, we examined how generous/stingy favor giving and matched favor giving shape employees' social status. Across two studies, we found that generous favor givers have higher social status than stingy givers in work groups. Matched favor giving, however, has a decreasingly positive curvilinear relationship with social status, in which the positive relationship between matched favor giving and social status tapers off after passing an optimal point. We also found that gratitude and perceived competence both mediate the relationship between generous/stingy favor giving and social status, while only perceived competence explains the curvilinear relationship between matched favor giving and social status.

\section{Theoretical Implications}

Our research has several theoretical implications. First, most researchers have used social exchange theory, which regards social status as an exchange resource for the received yet not reciprocated favors, to explain how employees gain social status through favor giving (e.g., Flynn, 2003; Flynn et al., 2006). Following the social-exchange logic, feelings of gratitude toward favor givers should explain the positive relationship between favor giving and status attainment. In the status-attainment literature, however, competence is a well-established route to status attainment, thus somewhat reflecting the cognitive side of social exchange, that the evaluation and judgment of key qualities of the exchange party relevant to task interactions will determine receivers' tendency to reciprocate received favors. Importantly, neither gratitude nor perceived competence has been clearly articulated or examined to explain the relationship between favor giving and social status. We have theorized and examined the gratitude and perceived competence mechanisms underlying different types of favor giving. Our findings indicate that both mechanisms work in generous/stingy favor giving, but only the perceived competence mechanism works in matched favor giving. 
Our dual-process model has theoretical implications for both social exchange theory and the social-status literature. On the one hand, the affective mechanism complements traditional social exchange theory, which over-emphasizes rational choice, regarding actors as unemotional (e.g., Cook \& Emerson, 1978; Lawler \& Thye, 1999; Molm \& Cook, 1995). We have demonstrated that gratitude, an emotion generated in social exchange, is important in determining people's behavior toward benefactors, particularly for those who owe to the other party. Moreover, we provide evidence of the emotion-related mechanism (i.e., gratitude) to ascend status hierarchy. This sheds light on a new route to social status beyond the well-known dominance and competence routes (e.g., Anderson \& Kilduff, 2009a; Bendersky \& Shah, 2012; Mazur, 1985; Mazur \& Booth, 1998). On the other hand, we showed that competence as an important characteristic is vital for people to judge the value of a potential partner, the utility of exchanging with the partner, and what kind of exchange can be made with the partner. Our findings thus concur with previous literature on social exchange that has emphasized a rational and cognitive micro-mediating mechanism (Lawler, 2001). Moreover, the mediating role of perceived competence in the curvilinear effect of matched favor giving on social status furthers the understanding of this competence route to status. The extent to which an actor is judged as competent via his or her favor giving is influenced by the distinctiveness of such behavior in the exchange network. We demonstrated that when others are able to display their continuous and increasing ability to return favors, it will dilute the focal actors' competence, thereby no longer driving further status conferral.

Second, the favor-exchange literature appears to focus more on studying "givers" and "takers" than on understanding "matchers," who actually inhabit the vast middle ground (Cain et al., 2014; Grant, 2013). It is surprising that, despite the ubiquity of matchers in organizations, we 
have little knowledge about how matchers obtain social status in work groups, for example, whether actors will gain ever-increasing status in matched favor giving as long as they keep giving favors. We investigated how both matched favor giving and generous/stingy favor giving shape group members' status attainment, providing a complete picture of how favor giving among coworkers influences the intragroup hierarchy. On the one hand, our findings validate previous research indicating that generosity leads to higher social status and stinginess leads to lower social status (e.g., Flynn, 2003; Flynn et al., 2006). On the other hand, different from what we usually presume - that people' social status will keep growing as long as they keep offering help to others, we have demonstrated that matched favor giving has a curvilinear relationship with social status. Our findings shed light on the importance of the distinctiveness of statusenhancing behavior and suggest that the significance and relevance of such behavior in promoting status could be diluted by the prevalence of others' similar behavior.

Third, our study has implications for the OCB and helping behavior literature. Most existing studies have focused on examining outcomes of OCB and helping behavior, particularly in regards to what supervisors or organizations can offer to the focal actor. Research findings, for example, have shown that employees are likely to receive higher performance ratings, organizational rewards, and career promotions via engaging in OCB and helping behavior (e.g., Allen \& Rush, 1998; Podsakoff et al., 1993; Seibert et al., 2001). The present study adopted the peers' perspective to investigate how helping behavior affects peer relationships and dynamics by examining social status, a type of peers' conferred rewards. This peer perspective on the social consequences of helping behavior nicely adds to an overall picture of the outcomes of employees' helping behavior or OCB.

\section{Practical Implications}


Our study also has several practical implications. First, social status usually brings a variety of benefits, and thus people generally strive for it. Before any social interactions take place, group members may have already distributed social status among members by simply assessing one another's status cues based on such "status characteristics" as gender, education, and ethnicity (Berger, Fisek, Norman, \& Zelditch, 1977; Magee \& Galinsky, 2008). Our findings suggest that individuals can climb the hierarchy ladder through giving favors to peers. Thus, behaviors such as favor giving can help individuals gain respect, influence, and social status, even though they may not possess those evident and predetermined "status cues."

Second, the overall positive relationship between matched favor giving and status attainment has important implications for reciprocal exchanges in the workplace. Reciprocity is an important norm of social exchange, and people are motivated to keep a balance between what they give and what they receive. Some may adhere to the reciprocity norm by always returning favors, while others may try to avoid receiving favors so as to free themselves from reciprocity obligations. Principles of fairness in social decision making suggest a person's behavior would be considered fair and equitable when one's contribution equals one's gain. Intuitively, a person who gives a lot but also receives a lot (i.e., high matched favor giving) should be seen as no different from another person who gives a little and also receives a little (i.e., low matched favor giving), as the transacted exchanges are equitable in both conditions. A high frequency of matched favor giving, however, is markedly different from low matched favor giving, in that individuals in the latter condition have much lower social status. Conventional wisdom suggests that giving favors enhances social status, whereas receiving favors hurts one's social status. Our findings, however, convey an important message that receiving does not necessarily inhibit status attainment as long as one can keep giving; "not giving" could inhibit status attainment even if 
one does not receive others' help. Thus, to ascend the status hierarchy, it is actively rendering favors to others, not avoiding receiving others' favors, that matters.

Third, our study has revealed a decreasingly positive relationship between matched favor giving and social status. Although group members generally reward individuals' favor-giving behavior with high social status, as suggested by the overall positive link between matched favor giving and status, such efforts to gain ever-increasing social status are in vain if individuals receive equivalent favors offered by their exchange parties. Given that favor giving consumes individuals' valuable but limited resources (Flynn, 2003; Grodal, Nelson, \& Siino, 2015), employees need to carefully manage their time and resources when offering favors to others.

\section{Limitations and Future Research Directions}

It is important to note several limitations in our study. First, although our findings are based on an experimental study and a multi-wave and multi-source field study, we cannot draw firm conclusions regarding causality. Theoretically, a reversed relationship, in which people with high status are more likely to give favors, makes sense, but empirical evidence has indicated instead that members who have lower status are actually more likely to help those who have higher status (Van der Vegt, Bunderson, \& Oosterhof, 2006). Moreover, the reversed curvilinear relationship between status and matched favor giving makes less sense. Nevertheless, we acknowledge the need for further evidence from more nuanced empirical assessments, such as those using event sampling methodology and panel analysis, before causality can be supported.

Second, we did not measure whether favors were "small" or "big" in terms of quality. For example, one major favor that satisfies an urgent need could be more helpful than several minor, less-urgent favors; thus a major favor may stimulate higher levels of the favor giver's perceived competence, as well as more gratitude and reciprocation (Flynn, 2006). Future studies might extend favor exchange research by investigating the role of the quality of favors. 
Third, our study found that matched favor giving has a curvilinear relationship with social status. Our interest centers on the overall amount of matched favor giving and how it affects social status. Matched favor giving, however, could have more nuanced patterns during the exchange process. The pattern of matched exchanges, for example, may be partly determined by who initiated the exchange and how receivers responded to the "first move." Specifically, in terms of reaping social status, there may be a difference between an individual who initiates the exchange by giving favors freely (i.e., first-mover) and an individual who tends to reciprocate favors given (i.e., second-mover). Indeed, research has suggested that employees who passively pay back received favors are likely to be seen as less altruistic or even self-serving (Cain et al., 2014). Thus, whether one is a proactive "first-mover" or a passive "second-mover" may have significant implications for status attainment.

We offer a few promising directions for future research to further explore the various forms of matched favor giving. First, future research could differentiate matched favor giving in terms of the motivation for favor giving. Actors in matched favor giving could be proactive "first-movers," who always initiate favor giving to others, or passive "second-movers," who always passively reciprocate others' favor giving, or "calculative agents," who carefully balance of the costs and benefits of every favor-giving behavior. "First-movers" appear to be more altruistic than both "second-movers" and "calculative agents," and, as a result, "first-movers" may have higher social status. Second, the scope of favor exchange might serve as a salient boundary condition of the link between favor giving and status attainment. For example, compared to one who only exchanges the same amount of favors with a few of his or her "insiders," an employee who evenly exchanges favors with a wide scope of his or her group members may be more likely to gain status in a work group. Third, time might serve as another parameter to examine different forms of matched favor giving. For example, in a single exchange of favor giving, "first-movers' may weigh heavier than "second-movers." Over a relatively long 
period of time, however, actors may switch back and forth between initiators and followers, and thus whether an employee is the "first-mover" may have less of an impact on status attainment.

Fourth, although we found essentially the same results regarding relationships between different types of favor giving and social status, and the same underlying mechanisms in both the Western context (Study 1) and Chinese context (Study 2), we are cautious about the cultural generalizability of our findings. In the Chinese context, for example, collectivistic norms might penalize "takers" more than in the Western context. Even though we have demonstrated that givers have higher status than takers in both cultural contexts, we still do not know whether takers have a worse condition in the Chinese context than in the Western context. Also, individuals in collectivist cultures may value collective goals more, and sometimes they need to subordinate personal pursuits to collective goals (e.g., Hofstede, 1991; Hofstede \& Peterson, 2000). Under such a condition, giving favors to other members may be a consequence of adhering to the collectivistic cultural norm. As a result, favor receivers may feel less grateful to receive favors. By contrast, in individualist cultures, people are expected to look after themselves. Giving favors to someone by using their own resources may be more likely to be seen as altruistic, thereby evoking more gratitude. Our findings, however, did not speak to such a nuance of potential cultural differences, and thus this topic awaits further research explorations.

Fifth, although we found that gratitude mediates the relationship between generous/stingy favor giving and social status, we acknowledge that other positive emotions may also underlie the relationship between favor giving and social status. For example, when an individual exhibits exceptional generosity to others, it may also induce feelings of admiration, a positive emotion generated by seeing extraordinary displays of skills, talents, or achievement (Algoe \& Haidt, 2009). Although empirical evidence tends to suggest that admiration makes people energized and motivated to work harder (Algoe \& Haidt, 2009), it is also possible that such a feeling may make people confer status to generous givers as a way to show appreciation and respect. Future 
research should further disentangle the mediating effects of gratitude from other types of positive emotions.

\section{Conclusion}

Our purpose for conducting this study was to complement the literature by examining the effects of generous/stingy and matched favor giving on social status, particularly the curvilinear effect of matched favor giving on status. By proposing a dual-process model, we found that gratitude and perceived competence explain the effect of generous/stingy favor giving on status, whereas only perceived competence underlies the link of matched favor giving and status. 


\section{References}

Aiken, L. S., \& West, S. G. (1991). Multiple regression: Testing and interpreting interactions. Newbury Park, CA: Sage.

Algoe, S. B. (2012). Find, remind, and bind: The functions of gratitude in everyday relationships. Social and Personality Psychology Compass, 6, 455-469. doi: 10.1111/j.17519004.2012.00439.x

Algoe, S. B., \& Haidt, J. (2009). Witnessing excellence in action: The 'other-praising' emotions of elevation, gratitude, and admiration. Journal of Positive Psychology, 4, 105-127. doi: $10.1080 / 17439760802650519$

Algoe, S. B., Haidt, J., \& Gable, S. L. (2008). Beyond reciprocity: Gratitude and relationships in everyday life. Emotion, 8, 425-429. doi: 10.1037/1528-3542.8.3.425

Allen, T. D., \& Rush, M. C. (1998). The effects of organizational citizenship behavior on performance judgments: A field study and a laboratory experiment. Journal of Applied Psychology, 83, 247-260. doi: 10.1037/0021-9010.83.2.247

Anderson, C., Hildreth, J. A. D., \& Howland, L. (2015). Is the desire for status a fundamental human motive? A review of the empirical literature. Psychological Bulletin, 141, 574-601. doi: $10.1037 / \mathrm{a} 0038781$

Anderson, C., John, O. P., Keltner, D., \& Kring, A. M. (2001). Who attains social status? Effects of personality and physical attractiveness in social groups. Journal of Personality and Social Psychology, 81, 116-132. doi: 10.1037/0022-3514.81.1.116

Anderson, C., \& Kilduff, G. J. (2009a). The pursuit of status in social groups. Current Directions in Psychological Science, 18, 295-298. doi: 10.1111/j.1467-8721.2009.01655.x

Anderson, C., \& Kilduff, G. J. (2009b). Why do dominant personalities attain influence in face- 
to-face groups? The competence-signaling effects of trait dominance. Journal of Personality and Social Psychology, 96, 491-503. doi: 10.1037/a0014201

Bai, F. (2016). Beyond dominance and competence: A moral virtue theory of status attainment. Personality and Social Psychology Review. Published online May 24, 2016. doi: $10.1177 / 1088868316649297$

Baumgarten-Tramer, F. (1938). "Gratefulness" in children and young people. The Pedagogical Seminary and Journal of Genetic Psychology, 53, 53-66. doi: $10.1080 / 08856559.1938 .10533797$

Bendersky, C., \& Shah, N. P. (2012). The cost of status enhancement: Performance effects of individuals' status mobility in task groups. Organization Science, 23, 308-322. doi: $10.1287 /$ orsc. 1100.0543

Berger, J., Cohen, B. P., \& Zelditch, M. (1972). Status characteristics and social interaction. American Sociological Review, 37, 241-255. doi: 10.2307/2093465

Berger, J., Fisek, M. H., Norman, R. Z., \& Zelditch, M. (1977). Status characteristics and social interaction: An expectation states approach. NY: Elsevier.

Berger, J., Rosenholtz, S. J., \& Zelditch, M. (1980). Status organizing processes. Annual Review of Sociology, 6, 479-508. doi: 10.1146/annurev.so.06.080180.002403

Blau, P. M. (1963). The dynamics of bureaucracy: A study of interpersonal relations in two government agencies. Chicago: University of Chicago Press.

Blau, P. M. (1964). Exchange and power in social life. New York: Wiley.

Brannon, L. A., \& Brock, T. C. (2001). Scarcity claims elicit extreme responding to persuasive messages: Role of cognitive elaboration. Personality and Social Psychology Bulletin, 27, 365-375. doi: $10.1177 / 0146167201273010$ 
Brislin, R., Lonner, W. J., \& Thorndike, R. (1973). Cross-cultural research methods. New York: Wiley.

Bunderson, J. S. (2003). Recognizing and utilizing expertise in work groups: A status characteristics perspective. Administrative Science Quarterly, 48, 557-591. doi: $10.2307 / 3556637$

Cain, D. M., Dana, J., \& Newman, G. E. (2014). Giving versus giving in. The Academy of Management Annals, 8, 505-533. doi: 10.1080/19416520.2014.911576

Casciaro, T., Gino, F., \& Kouchaki, M. (2014). The contaminating effects of building instrumental ties how networking can make us feel dirty. Administrative Science Quarterly, 59, 705-735. doi: 10.1177/0001839214554990

Cheng, B. S., Chou, L. F., Wu, T. Y., Huang, M. P., \& Farh, J. L. (2004). Paternalistic leadership and subordinate responses: Establishing a leadership model in Chinese organizations. Asian Journal of Social Psychology, 7, 89-117. doi: 10.1111/j.1467-839x.2004.00137.x

Cheng, J. T., Tracy, J. L., Foulsham, T., Kingstone, A., \& Henrich, J. (2013). Two ways to the top: Evidence that dominance and prestige are distinct yet viable avenues to social rank and influence. Journal of Personality and Social Psychology, 104, 103-125. doi:

$10.1037 / \mathrm{a} 0030398$

Cook, K. S., \& Emerson, R. M. (1978). Power, equity, and commitment in exchange networks. American Sociological Review, 43, 721-739. doi: 10.2307/2094546

Ditto, P. H., \& Jemmott, J. B. (1989). From rarity to evaluative extremity: Effects of prevalence information on evaluations of positive and negative characteristics. Journal of Personality and Social Psychology, 57, 16-26. doi: 10.1037//0022-3514.57.1.16

Edwards, J. R., \& Cable, D. M. (2009). The value of value congruence. Journal of Applied 
Psychology, 94, 654-677. doi: 10.1037/a0014891

Edwards, J. R., \& Parry, M. E. (1993). On the use of polynomial regression equations as an alternative to difference scores in organizational research. Academy of Management Journal, 36, 1577-1613. doi: 10.2307/256822

Edwards, J. R., \& Rothbard, N. P. (1999). Work and family stress and well-being: An examination of person-environment fit in the work and family domains. Organizational Behavior and Human Decision Processes, 77, 85-129. doi: 10.1006/obhd.1998.2813

Ehrhart, M. G., Bliese, P. D., \& Thomas, J. L. (2006). Unit-level OCB and unit effectiveness: Examining the incremental effect of helping behavior. Human Performance, 19, 159-173. doi: 10.1207/s15327043hup1902_4

Fisher, J. D., Nadler, A., \& Whitcher-Alagna, S. (1982). Recipient reactions to aid. Psychological Bulletin, 91, 27-54. doi: 10.1037/0033-2909.91.1.27

Flanagan, J. C. (1954). The critical incident technique. Psychological Bulletin, 51, 327-358. doi: $10.1037 / \mathrm{h} 0061470$

Flynn, F. J. (2003). How much should I give and how often? The effects of generosity and frequency of favor exchange on social status and productivity. Academy of Management Journal, 46, 539-553. doi: 10.2307/30040648

Flynn, F. J. (2006). How much is it worth to you? Subjective evaluations of help in organizations. Research in Organizational Behavior, 27, 133-174. doi: 10.1016/s01913085(06)27004-7

Flynn, F. J., Reagans, R. E., Amanatullah, E. T., \& Ames, D. R. (2006). Helping one's way to the top: Self-monitors achieve status by helping others and knowing who helps whom. Journal of Personality and Social Psychology, 91, 1123-1137. doi: 10.1037/0022-3514.91.6.1123 
Foa, E. B., \& Foa, U. G. (1980). Resource theory: Interpersonal behavior as exchange. In K. J. Gergen, M. S. Greenberg, \& R. H. Willis (Eds.), Social exchange: Advances in theory and research (pp. 77-94). New York: Plenum Press.

Foa, U. G., \& Foa, E. B. (1974). Societal structures of the mind. Springfield, IL: Thomas.

Gouldner, A. W. (1960). The norm of reciprocity: A preliminary statement. American Sociological Review, 25, 161-178. doi: 10.2307/2092623

Grant, A. M. (2013). Give and take: A revolutionary approach to success. Penguin Group.

Grodal, S., Nelson, A. J., \& Siino, R. M. (2015). Help-seeking and help-giving as an organizational routine: Continual engagement in innovative work. Academy of Management Journal, 58, 136-168. doi: 10.5465/amj.2012.0552

Hardy, C. L., \& Van Vugt, M. (2006). Nice guys finish first: The competitive altruism hypothesis. Personality and Social Psychology Bulletin, 32, 1402-1413. doi: $10.1177 / 0146167206291006$

Hayes, A. F., \& Preacher, K. J. (2010). Quantifying and testing indirect effects in simple mediation models when the constituent paths are nonlinear. Multivariate Behavioral Research, 45, 627-660. doi: 10.1080/00273171.2010.498290

Heise, D. R. (1972). Employing nominal variables, induced variables, and block variables in path analyses. Sociological Methods \& Research, 1, 147-173. doi: $10.1177 / 004912417200100201$

Hofstede, G. (1991). Cultures and organizations: Software of the mind. London: McGraw-Hill. Hofstede, G., \& Peterson, M. F. (2000). Culture: National values and organizational practices. In N. M. Ashkanasy, C. P. Wilderom, \& M. F. Peterson (Eds.), Handbook of organizational culture and climate (pp. 401-416). Thousand Oaks, CA: Sage. 
Jansen, K. J., \& Kristof-Brown, A. L. (2005). Marching to the beat of a different drummer: Examining the impact of pacing congruence. Organizational Behavior and Human Decision Processes, 97, 93-105. doi: 10.1016/j.obhdp.2005.03.005

Kalma, A. P., Visser, L., \& Peeters, A. (1993). Sociable and aggressive dominance: Personality differences in leadership style? Leadership Quarterly, 4, 45-64. doi: 10.1016/10489843(93)90003-c

Keltner, D., Gruenfeld, D. H., \& Anderson, C. (2003). Power, approach, and inhibition. Psychological Review, 110, 265-284. doi: 10.1037/0033-295x.110.2.265

Lawler, E. J. (2001). An affect theory of social exchange. American Journal of Sociology, 107, 321-352. doi: $10.1086 / 324071$

Lawler, E. J., \& Thye, S. R. (1999). Bringing emotions into social exchange theory. Annual Review of Sociology, 25, 217-244. doi: 10.1146/annurev.soc.25.1.217

Lawler, E. J., Thye, S. R., \& Yoon, J. (2000). Emotion and group cohesion in productive exchange. American Journal of Sociology, 106, 616-657. doi: 10.1086/318965

Lee, K., \& Allen, N. J. (2002). Organizational citizenship behavior and workplace deviance: The role of affect and cognitions. Journal of Applied Psychology, 87, 131-142. doi: $10.1037 / 0021-9010.87 .1 .131$

Liang, L. H., Lian, H., Brown, D. J., Ferris, D. L., Hanig, S., \& Keeping, L. M. (2016). Why are abusive supervisors abusive? A dual-system self-control model. Academy of Management Journal, 59, 1385-1406. doi: 10.5465/amj.2014.0651

Magee, J. C., \& Galinsky, A. D. (2008). Social hierarchy: The self-reinforcing nature of power and status. The Academy of Management Annals, 2, 351-398. doi: $10.1080 / 19416520802211628$ 
Matta, F. K., Scott, B. A., Koopman, J., \& Conlon, D. E. (2014). Does seeing "eye to eye" affect work engagement and organizational citizenship behavior? A role theory perspective on LMX agreement. Academy of Management Journal, 58, 1686-1708. doi: 10.5465/amj.2014.0106

Mayer, D. M., Greenbaum, R. L., Kuenzi, M., \& Shteynberg, G. (2009). When do fair procedures not matter? A test of the identity violation effect. Journal of Applied Psychology, 94, 142161. doi: $10.1037 / \mathrm{a} 0013108$

Mazur, A. (1985). A biosocial model of status in face-to-face primate groups. Social Forces, 64, 377-402. doi: 10.1093/sf/64.2.377

Mazur, A., \& Booth, A. (1998). Testosterone and dominance in men. Behavioral and Brain Sciences, 21, 353-397. doi: 10.1017/s0140525x98001228

McCullough, M. E., Kilpatrick, S. D., Emmons, R. A., \& Larson, D. B. (2001). Is gratitude a moral affect? Psychological Bulletin, 127, 249-266. doi: 10.1037/0033-2909.127.2.249

Meade, A. W., \& Craig, S. B. (2012). Identifying careless responses in survey data. Psychological Methods, 17, 437-455. doi: 10.1037/e518362013-127

Molm, L. D., \& Cook, K. S. (1995). Social exchange and exchange networks. In K. S. Cook, G. A. Fine, \& J. S. House (Eds.), Sociological perspectives on social psychology (pp. 209235). Boston: Allyn \& Bacon.

Motowidlo, S. J., \& Van Scotter, J. R. (1994). Evidence that task performance should be distinguished from contextual performance. Journal of Applied Psychology, 79, 475-480. doi: $10.1037 / 0021-9010.79 .4 .475$

Ostroff, C., Atwater, L. E., \& Feinberg, B. J. (2004). Understanding self-other agreement: A look at rater and ratee characteristics, context, and outcomes. Personnel Psychology, 57, 333- 
375. doi: 10.1111/j.1744-6570.2004.tb02494.x

Pettigrew, T. F. (1967). Social evaluation theory: Convergences and applications. In D. Levine (Ed.), Nebraska symposium on motivation (Vol. 15, pp. 241-311). Lincoln: University of Nebraska Press.

Podsakoff, P. M., Ahearne, M., \& MacKenzie, S. B. (1997). Organizational citizenship behavior and the quantity and quality of work group performance. Journal of Applied Psychology, 82, 262-270. doi: 10.1037//0021-9010.82.2.262

Podsakoff, P. M., MacKenzie, S. B., \& Hui, C. (1993). Organizational citizenship behaviors and managerial evaluations of employee performance: A review and suggestions for future research. In G. R. Ferris (Ed.), Research in personnel and human resources management (Vol. 11, pp. 1-40). Greenwich, CT: JAI Press.

Preacher, K. J., \& Hayes, A. F. (2008). Asymptotic and resampling strategies for assessing and comparing indirect effects in multiple mediator models. Behavior Research Methods, 40, 879-891. doi: 10.3758/brm.40.3.879

Preacher, K. J., \& Selig, J. P. (2012). Advantages of Monte Carlo confidence intervals for indirect effects. Communication Methods and Measures, 6, 77-98. doi: $10.1080 / 19312458.2012 .679848$

Ridgeway, C. L. (2001). Gender, status, and leadership. Journal of Social Issues, 57, 637-655. doi: $10.1111 / 0022-4537.00233$

Ridgeway, C. L., \& Berger, J. (1986). Expectations, legitimation, and dominance behavior in task groups. American Sociological Review, 51, 603-617. doi: 10.2307/2095487

Ross, M., \& Sicoly, F. (1979). Egocentric biases in availability and attribution. Journal of Personality and Social Psychology, 37, 322-336. doi: 10.1037//0022-3514.37.3.322 
Schaubroeck, J., Lam, S. S., \& Peng, A. C. (2011). Cognition-based and affect-based trust as mediators of leader behavior influences on team performance. Journal of Applied Psychology, 96, 863-871. doi: 10.1037/a0022625

Seibert, S. E., Kraimer, M. L., \& Crant, J. M. (2001). What do proactive people do? A longitudinal model linking proactive personality and career success. Personnel Psychology, 54, 845-874. doi: 10.1111/j.1744-6570.2001.tb00234.x

Selig, J. P., \& Preacher, K. J. (2008). Monte Carlo method for assessing mediation: An interactive tool for creating confidence intervals for indirect effects [Computer software]. Available from http://quantpsy.org/.

Shanock, L. R., Baran, B. E., Gentry, W. A., Pattison, S. C., \& Heggestad, E. D. (2010). Polynomial regression with response surface analysis: A powerful approach for examining moderation and overcoming limitations of difference scores. Journal of Business and Psychology, 25, 543-554. doi: 10.1007/s10869-010-9183-4

Simmel, G. (1950). The sociology of Georg Simmel. Glencoe, IL: Free Press.

Smith, E. A., \& Bird, R. L. B. (2000). Turtle hunting and tombstone opening: Public generosity as costly signaling. Evolution and Human Behavior, 21, 245-261. doi: 10.1016/s1090$5138(00) 00031-3$

Snijders, T. A. B. \& Bosker, R. J. (1999). Multilevel analysis: An introduction to basic and advanced multilevel modeling. Thousand Oaks, CA: Sage.

Spence, J. R., Brown, D. J., Keeping, L. M., \& Lian, H. (2014). Helpful today, but not tomorrow? Feeling grateful as a predictor of daily organizational citizenship behaviors. Personnel Psychology, 67, 705-738. doi: 10.1111/peps.12051

Stone, D. L., \& Stone, E. F. (1985). The effects of feedback consistency and feedback favorability on self-perceived task competence and perceived feedback accuracy. 
Organizational Behavior and Human Decision Processes, 36, 167-185. doi: 10.1016/0749$5978(85) 90011-1$

Van der Vegt, G. S., Bunderson, J. S., \& Oosterhof, A. (2006). Expertness diversity and interpersonal helping in teams: Why those who need the most help end up getting the least. Academy of Management Journal, 49, 877-893. doi: 10.5465/amj.2006.22798169

Warner, R. M., Kenny, D. A., \& Stoto, M. (1979). A new round robin analysis of variance for social interaction data. Journal of Personality and Social Psychology, 37, 1742-1757. doi: $10.1037 / / 0022-3514.37 .10 .1742$

Wellman, N., Mayer, D. M., Ong, M., \& DeRue, D. S. (2016). When are do-gooders treated badly? Legitimate power, role expectations, and reactions to moral objection in organizations. Journal of Applied Psychology, 101, 793-814. doi: 10.1037/ap10000094

Wojciszke, B., Abele, A. E., \& Baryla, W. (2009). Two dimensions of interpersonal attitudes: Liking depends on communion, respect depends on agency. European Journal of Social Psychology, 39, 973-990. doi: 10.1002/ejsp.595

Zhang, Z., Wang, M., \& Shi, J. (2012). Leader-follower congruence in proactive personality and work outcomes: The mediating role of leader-member exchange. Academy of Management Journal, 55, 111-130. doi: 10.5465/amj.2009.0865 


\section{Table 1}

Results of Independent-Samples T-Tests for Favor Giving Conditions, Study 1

\begin{tabular}{|c|c|c|c|c|c|c|c|}
\hline Type & & Condition 1 & Condition 2 & Condition 3 & $\begin{array}{c}\text { Condition } 1 \text { versus } \\
\text { Condition } 2\end{array}$ & $\begin{array}{c}\text { Condition } 1 \text { versus } \\
\text { Condition } 3\end{array}$ & $\begin{array}{l}\text { Condition } 2 \text { versus } \\
\text { Condition } 3\end{array}$ \\
\hline \multirow{4}{*}{$\begin{array}{c}\text { Generous/Stingy } \\
\text { favor giving }\end{array}$} & Gratitude & $\begin{array}{r}M=3.27 \\
(S D=1.02)\end{array}$ & $\begin{array}{c}M=4.14 \\
(S D=.48)\end{array}$ & $\begin{array}{c}M=4.60 \\
(S D=.43)\end{array}$ & $\begin{array}{c}\mathrm{t}(85)=4.78, p=.000 \\
d=.87\end{array}$ & $\begin{array}{c}\mathrm{t}(86)=7.52, p=.000 \\
d=1.33\end{array}$ & $\begin{array}{c}\mathrm{t}(73)=4.40, p=.000 \\
d=.46\end{array}$ \\
\hline & Perceived competence & $\begin{array}{r}M=3.17 \\
(S D=1.02)\end{array}$ & $\begin{array}{c}M=4.46 \\
(S D=.57)\end{array}$ & $\begin{array}{c}M=4.87 \\
(S D=.29)\end{array}$ & $\begin{array}{c}\mathrm{t}(85)=6.91, p=.000 \\
d=1.29\end{array}$ & $\begin{array}{c}\mathrm{t}(86)=9.97, p=.000 \\
d=1.70\end{array}$ & $\begin{array}{c}\mathrm{t}(73)=3.92, p=.000 \\
d=.41\end{array}$ \\
\hline & \multirow[t]{2}{*}{ Social status } & $\begin{array}{r}M=3.09 \\
(S D=1.16)\end{array}$ & $\begin{array}{c}M=4.10 \\
(S D=.48)\end{array}$ & $\begin{array}{c}M=4.68 \\
(S D=.35)\end{array}$ & $\begin{array}{c}\mathrm{t}(85)=4.97, p=.000 \\
d=1.01\end{array}$ & $\begin{array}{c}\mathrm{t}(86)=8.82, p=.000 \\
d=1.59\end{array}$ & $\begin{array}{c}\mathrm{t}(73)=6.09, p=.000 \\
d=.58\end{array}$ \\
\hline & & Condition 4 & Condition 5 & Condition 6 & $\begin{array}{c}\text { Condition } 4 \text { versus } \\
\text { Condition } 5\end{array}$ & $\begin{array}{c}\text { Condition } 4 \text { versus } \\
\text { Condition } 6\end{array}$ & $\begin{array}{l}\text { Condition } 5 \text { versus } \\
\text { Condition } 6\end{array}$ \\
\hline \multirow{3}{*}{$\begin{array}{c}\text { Matched } \\
\text { favor giving }\end{array}$} & Gratitude & $\begin{array}{l}M=4.48 \\
(S D=.56)\end{array}$ & $\begin{array}{c}M=4.52 \\
(S D=.43)\end{array}$ & $\begin{array}{c}M=4.57 \\
(S D=.45)\end{array}$ & $\begin{array}{c}\mathrm{t}(81)=.33, p=.744 \\
d=.04\end{array}$ & $\begin{array}{c}\mathrm{t}(84)=.79, p=.435 \\
d=.09\end{array}$ & $\begin{array}{c}\mathrm{t}(79)=.51, p=.611 \\
d=.05\end{array}$ \\
\hline & Perceived competence & $\begin{array}{l}M=4.45 \\
(S D=.61)\end{array}$ & $\begin{array}{c}M=4.73 \\
(S D=.34)\end{array}$ & $\begin{array}{c}M=4.70 \\
(S D=.37)\end{array}$ & $\begin{array}{c}\mathrm{t}(81)=2.46, p=.016 \\
d=.28\end{array}$ & $\begin{array}{c}\mathrm{t}(84)=2.22 p=.029 \\
d=.25\end{array}$ & $\begin{array}{c}\mathrm{t}(79)=.35, p=.726 \\
d=.03\end{array}$ \\
\hline & Social status & $\begin{array}{l}M=4.19 \\
(S D=.71)\end{array}$ & $\begin{array}{l}M=4.48 \\
(S D=.47)\end{array}$ & $\begin{array}{l}M=4.52 \\
(S D=.45)\end{array}$ & $\begin{array}{c}\mathrm{t}(81)=2.18, p=.032 \\
d=.29\end{array}$ & $\begin{array}{c}\mathrm{t}(84)=2.57, p=.012 \\
d=.33\end{array}$ & $\begin{array}{c}\mathrm{t}(79)=.37, p=.716 \\
d=.04\end{array}$ \\
\hline
\end{tabular}

Note. Condition $1=$ Least generous favor giving $(N=50)$; Condition $2=$ Medium generous favor giving $(N=37)$; Condition $3=$ High generous favor giving $(N=38)$;

Condition $4=$ Low matched favor giving $(N=44)$; Condition $5=$ Medium matched favor giving $(N=39)$; Condition $6=$ High matched favor giving $(N=42)$. 


\section{Table 2}

Means, Standard Deviations, and Correlations among Key Variables, Study 2

\begin{tabular}{|c|c|c|c|c|c|c|c|c|c|c|c|c|c|}
\hline Variable & $M$ & $S D$ & 1 & 2 & 3 & 4 & 5 & 6 & 7 & 8 & 9 & 10 & 11 \\
\hline 1. Gender & 0.29 & 0.46 & & & & & & & & & & & \\
\hline 2. Age (Year) & 33.59 & 9.78 & .00 & & & & & & & & & & \\
\hline 3. Education & 3.16 & 0.99 & $.19 * * *$ & $.37 * * *$ & & & & & & & & & \\
\hline 4. Tenure (Year) & 11.02 & 7.50 & $.15 * *$ & $.62 * * *$ & $.33 * * *$ & & & & & & & & \\
\hline 5. Organization type & 0.61 & 0.49 & $.41 * * *$ & $.26 * * *$ & $.73 * * *$ & $.42 * * *$ & & & & & & & \\
\hline 6. Job performance (Time 1) & 4.54 & 1.33 & $.23 * * *$ & $.12 *$ & $.45 * * *$ & $.20 * * *$ & $.64 * * *$ & & & & & & \\
\hline 7. Group-level favor receiving (Time 1) & 2.74 & 0.53 & $.12 *$ & $.26 * * *$ & $.40 * * *$ & $.21 * * *$ & $.41 * * *$ & $.25 * * *$ & & & & & \\
\hline 8. Favor giving (Time 1) & 2.76 & 0.68 & .04 & $.16^{* *}$ & $.29 * * *$ & $.18 * *$ & $.31 * * *$ & $.17 * *$ & $.75 * * *$ & & & & \\
\hline 9. Favor receiving (Time 1) & 2.71 & 0.86 & .00 & $.26^{* * *}$ & $.31 * * *$ & $.13^{*}$ & $.27 * * *$ & $.19 * * *$ & $.62 * * *$ & $.27 * * *$ & & & \\
\hline 10. Gratitude (Time 1) & 3.76 & 0.49 & -.08 & .04 & -.01 & -.01 & -.09 & -.06 & $.24 * * *$ & $.35 * * *$ & .02 & & \\
\hline 11. Perceived competence (Time 1) & 3.92 & 0.54 & -.05 & $.11^{*}$ & .08 & .03 & -.04 & .01 & $.37 * * *$ & $.42 * * *$ & $.17^{* *}$ & $.58 * * *$ & \\
\hline 12. Social status (Time 2) & 4.48 & 0.86 & $.17 * *$ & $.23 * * *$ & $.52 * * *$ & $.29 * * *$ & $.61 * * *$ & $.45 * * *$ & $.49 * * *$ & $.54 * * *$ & $.25 * * *$ & $.39 * * *$ & $.56 * * *$ \\
\hline
\end{tabular}

Note. $\mathrm{N}=362$.

Gender: Female $=0$, Male $=1$. Education: Junior high school or below $=1$, High school or equivalent $=2$, College or associate's degree $=3$, Bachelor degree or above $=4$, Other $=5$. Organization type: Kindergarten $=0$, Telecommunications company $=1$.

$* p<.05 . * * p<.01 . * * *<.001$. 
Cross-Level Polynomial Regressions of Perceived Competence, Gratitude, and Social Status on Favor Giving, Study 2

\begin{tabular}{|c|c|c|c|c|}
\hline \multirow{2}{*}{ Variables } & \multirow{2}{*}{$\frac{\text { Perceived competence }}{\text { Model } 1}$} & \multirow{2}{*}{$\begin{array}{c}\text { Gratitude } \\
\text { Model } 2\end{array}$} & \multicolumn{2}{|c|}{ Social status } \\
\hline & & & Model 3 & Model 4 \\
\hline Constant & $0.05(.21)$ & $-0.07(.20)$ & $-0.03(.20)$ & $-0.05(.14)$ \\
\hline Group-level favor receiving & $0.01(.08)$ & $-0.02(.09)$ & $-0.05(.08)$ & $-0.05(.06)$ \\
\hline Gender & $0.02(.10)$ & $0.02(.09)$ & $-0.09(.10)$ & $-0.10(.07)$ \\
\hline Age & $0.00(.01)$ & $0.00(.01)$ & $0.00(.01)$ & $-0.00(.00)$ \\
\hline Education & $0.06(.07)$ & $0.05(.06)$ & $0.07(.06)$ & $0.03(.04)$ \\
\hline Tenure & $-0.00(.01)$ & $-0.00(.01)$ & $0.01(.01)$ & $0.01(.01)$ \\
\hline Organization type & $-0.26(.18)$ & $-0.18(.19)$ & $-0.34(.18)$ & $-0.17(.13)$ \\
\hline Job performance & $0.01(.06)$ & $-0.04(.05)$ & $0.07(.05)$ & $0.07(.04)$ \\
\hline Favor giving (FG) & $0.39 * * *(.06)$ & $0.36^{* * *}(.06)$ & $0.45 * * *(.06)$ & $0.15 * *(.05)$ \\
\hline Favor receiving $(\mathrm{FR})$ & $0.01(.06)$ & $-0.06(.05)$ & $-0.01(.05)$ & $0.00(.04)$ \\
\hline $\mathrm{FG}^{2}$ & $-0.00(.03)$ & $-0.01(.04)$ & $-0.01(.04)$ & $-0.00(.03)$ \\
\hline $\mathrm{FG} * \mathrm{FR}$ & $-0.05(.04)$ & $0.00(.05)$ & $-0.11 *(.05)$ & $-0.08 * *(.03)$ \\
\hline $\mathrm{FR}^{2}$ & $-0.07 *(.03)$ & $-0.02(.03)$ & $-0.06^{*}(.03)$ & $-0.01(.02)$ \\
\hline Perceived competence & - & - & - & $0.59 * * *(.04)$ \\
\hline Gratitude & - & - & - & $0.18 * * *(.04)$ \\
\hline $\mathrm{R}^{2}$ & .27 & .16 & .28 & .64 \\
\hline$\Delta \mathrm{R}^{2}$ & - & - & - & .36 \\
\hline \multicolumn{5}{|l|}{ Matched $(F G=F R)$ line } \\
\hline Slope & $.40 * * *(.08)$ & $.30 * * *(.08)$ & $.44^{* * *}(.08)$ & $.15^{*}(.06)$ \\
\hline Curvature & $-.12 *(.06)$ & $-.03(.07)$ & $-.18 * *(.07)$ & $-.09(.05)$ \\
\hline \multicolumn{5}{|l|}{ Generous/Stingy $(F G=-F R)$ line } \\
\hline Slope & $.38 * * *(.08)$ & $.42 * * *(.08)$ & $.46 * * *(.08)$ & $.15 *(.06)$ \\
\hline Curvature & $-.02(.06)$ & $-.03(.07)$ & $.04(.07)$ & $.07(.05)$ \\
\hline $\mathrm{F}$ for the 3 quadratic terms & $5.35^{*}$ & 0.66 & $8.44 *$ & $6.10^{*}$ \\
\hline
\end{tabular}

Note. $\mathrm{N}=362$. Standardized regression coefficients are reported. $\mathrm{R}^{2}$ was calculated according to Snijders and Bosker (1999, $\left.\mathrm{p} .102\right)$. $\Delta \mathrm{R}^{2}$ refers to the change in explained variance attributable to the inclusion of perceived competence and gratitude. Standard errors are presented in parentheses.

$* p<.05$. ** $p<.01 . * * * p<.001$. 
Results for Indirect Effects of Favor Giving on Social Status, Study 2

\begin{tabular}{|c|c|c|c|}
\hline Variables & $\begin{array}{l}\text { Perceived } \\
\text { competence }\end{array}$ & Gratitude & Social status \\
\hline $\begin{array}{l}\text { Coefficient of the block variable } \\
\text { (i.e., direct effect of generous/stingy/matched favor giving) }\end{array}$ & $.50 * * *(.08)$ & $.49 * * *(.07)$ & $.25 * * *(.07)$ \\
\hline Coefficient of perceived competence $\left(\gamma_{\text {perceived competence }}\right)$ & - & - & $.44 * * *(.03)$ \\
\hline Coefficient of gratitude ( $\left.\gamma_{\text {gratitude }}\right)$ & - & - & $.13 * * *(.03)$ \\
\hline $\begin{array}{l}\text { Indirect effect via perceived competence } \\
\qquad\left(=.50 * \gamma_{\text {perceived competence }}\right)\end{array}$ & - & - & $.22 * * *$ \\
\hline 95\% Monte Carlo confidence interval for the indirect effect & - & - & {$[.15, .30]$} \\
\hline $\begin{array}{l}\text { Indirect effect via gratitude } \\
\qquad\left(=.49 * \gamma_{\text {gratitude }}\right)\end{array}$ & - & - & $.06^{* *}$ \\
\hline 95\% Monte Carlo confidence interval for the indirect effect & - & - & {$[.03, .10]$} \\
\hline
\end{tabular}

Note. Standardized regression coefficients are reported. Standard errors are presented in parentheses.

$* p<.05 . * * p<.01 . * * * p<.001$. 


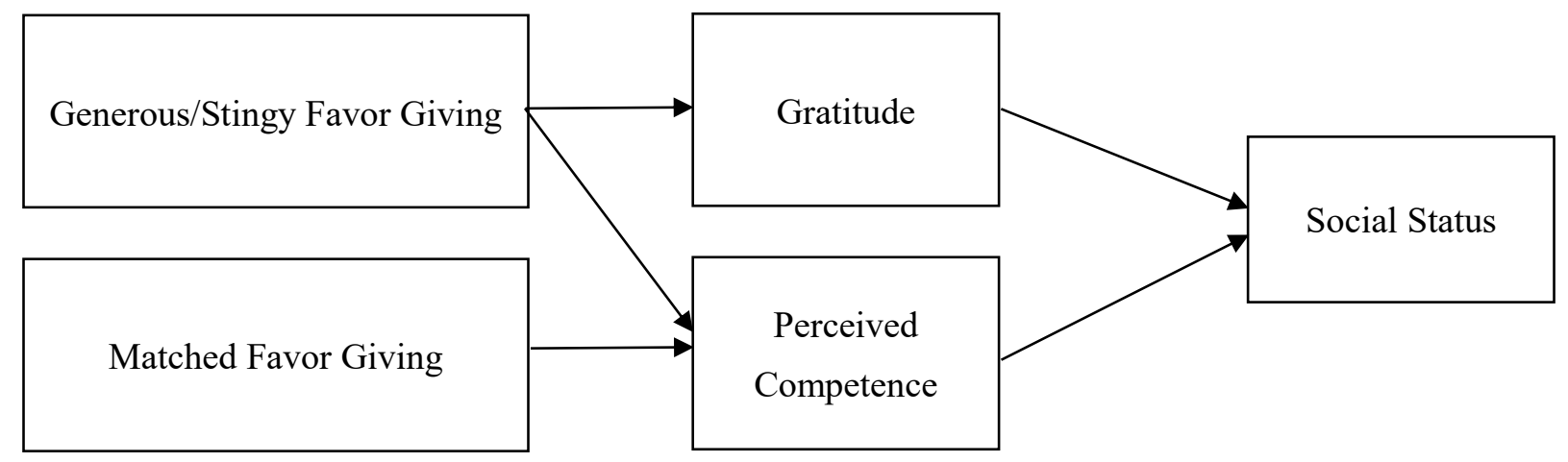

Figure 1. Research model. 


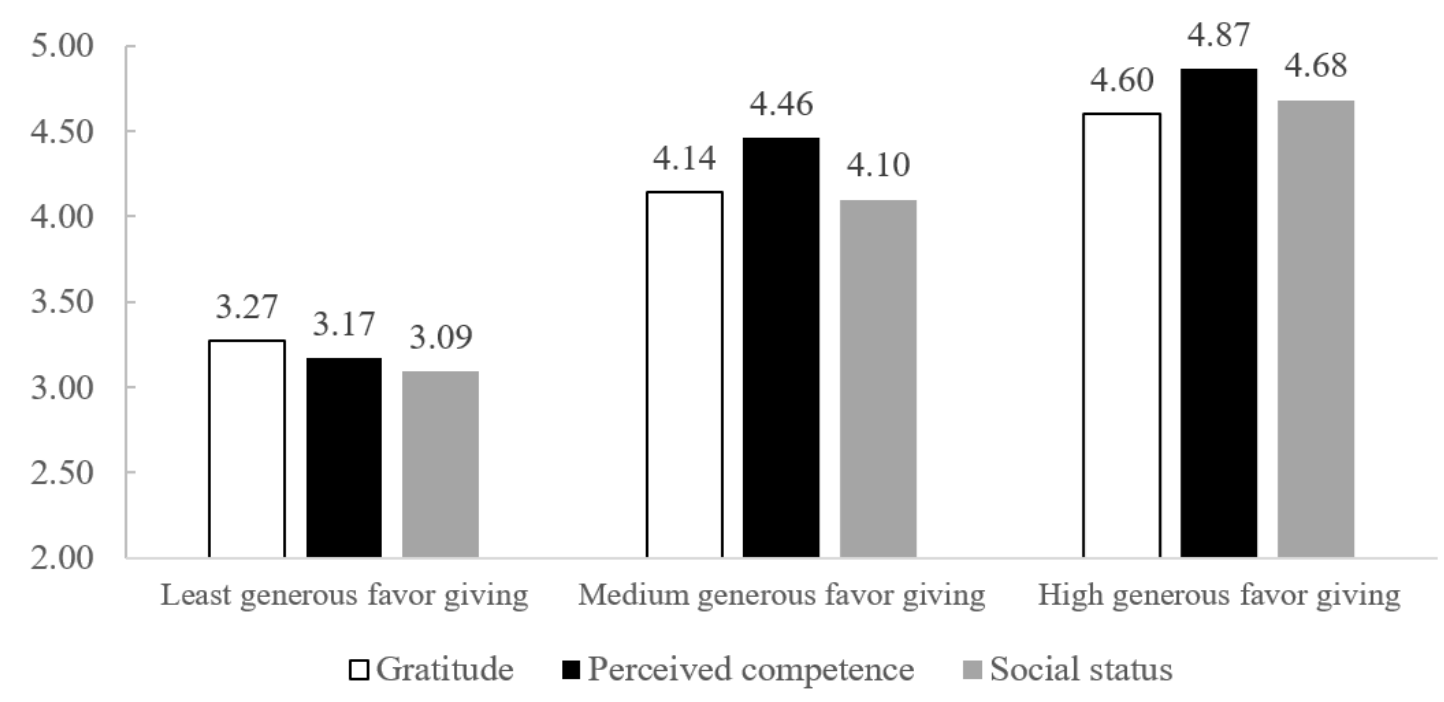

Figure 2. Generous/stingy favor giving, gratitude, perceived competence, and social status, Study 1. 


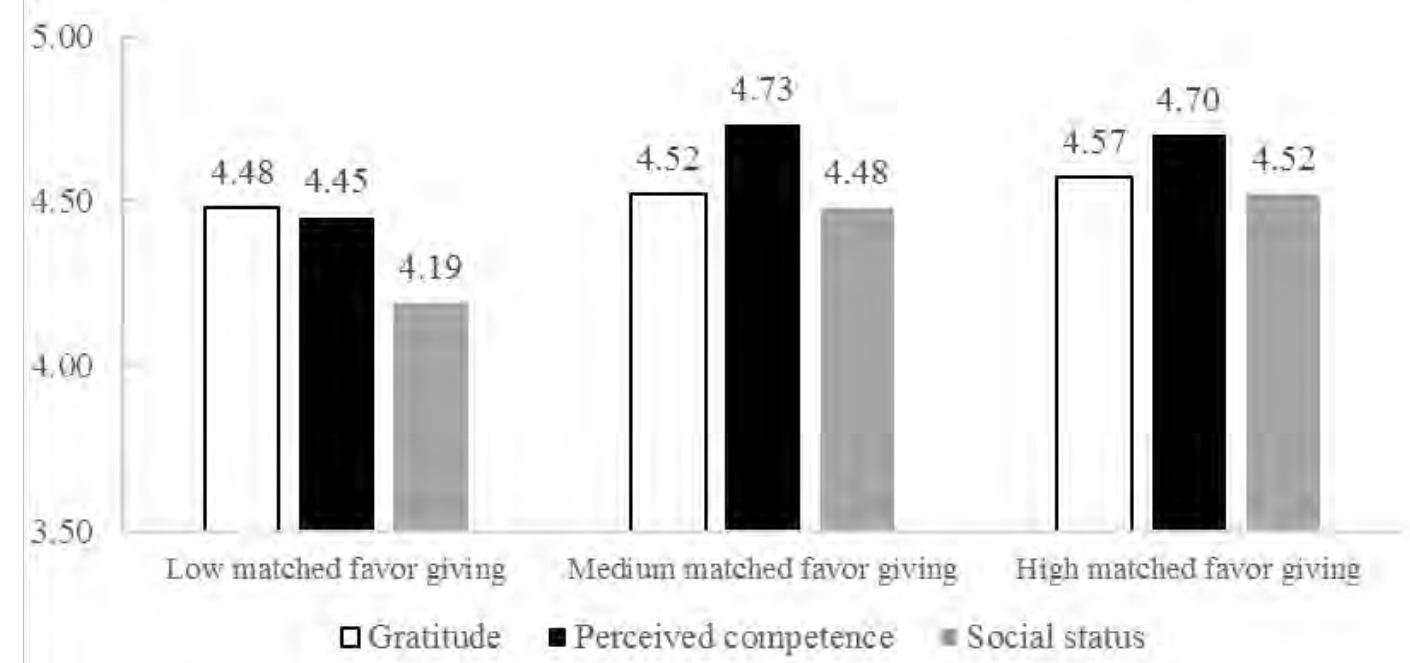

Figure 3. Matched favor giving, gratitude, perceived competence, and social status, Study 1. 
$-2$

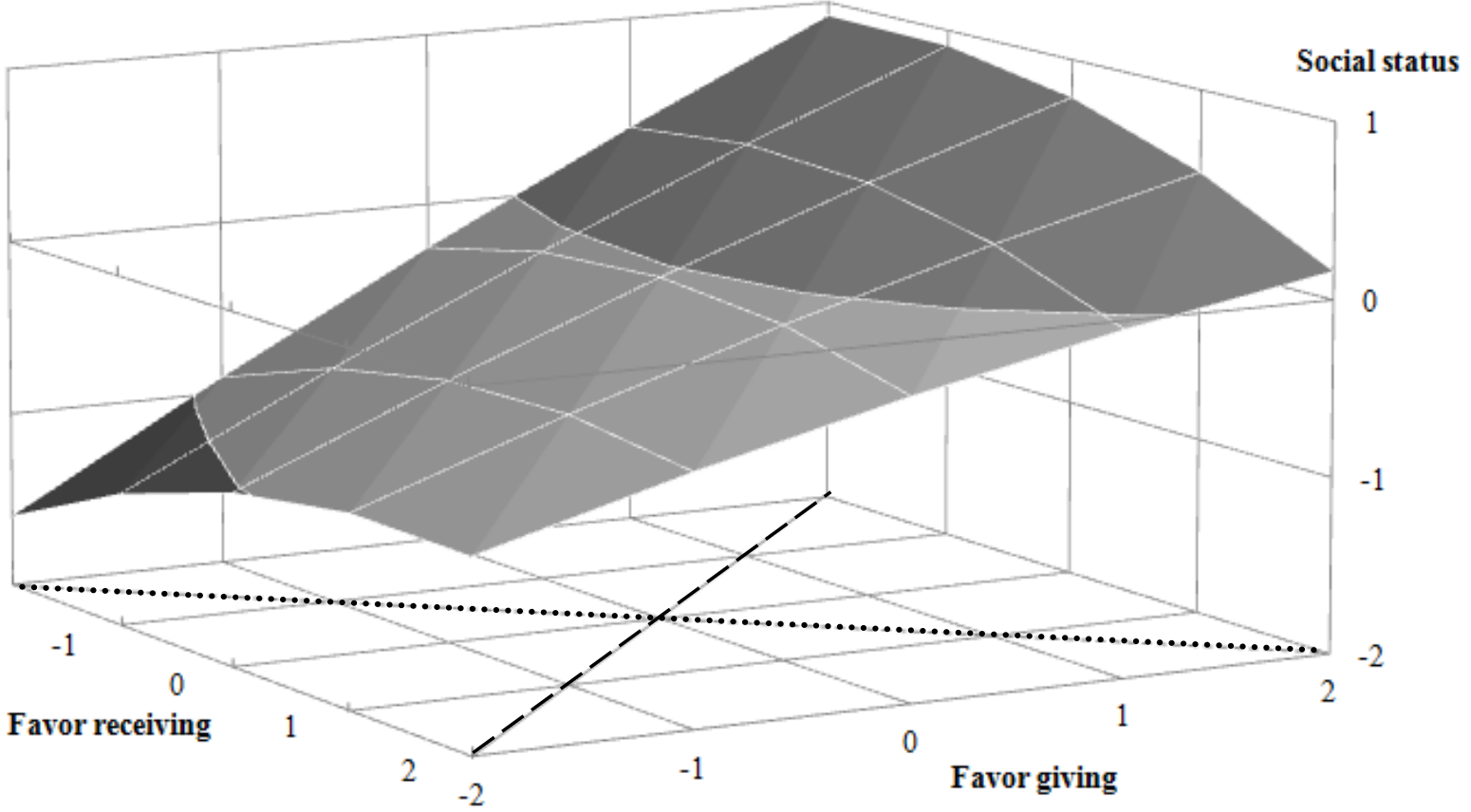

Figure 4. Effects of generous, stingy, and matched favor giving on social status, Study 2. Following Zhang and colleagues (2012), for better visual examination, we changed the orientation in the response surface from what Edwards and Parry (1993) used. The long-dashed line represents the generous/stingy line, along which favor giving becomes more generous from the front corner to the rear corner; the round-dotted line represents the matched line, along which the frequency of matched favor giving increases from the left corner to the right corner. 
$-2$

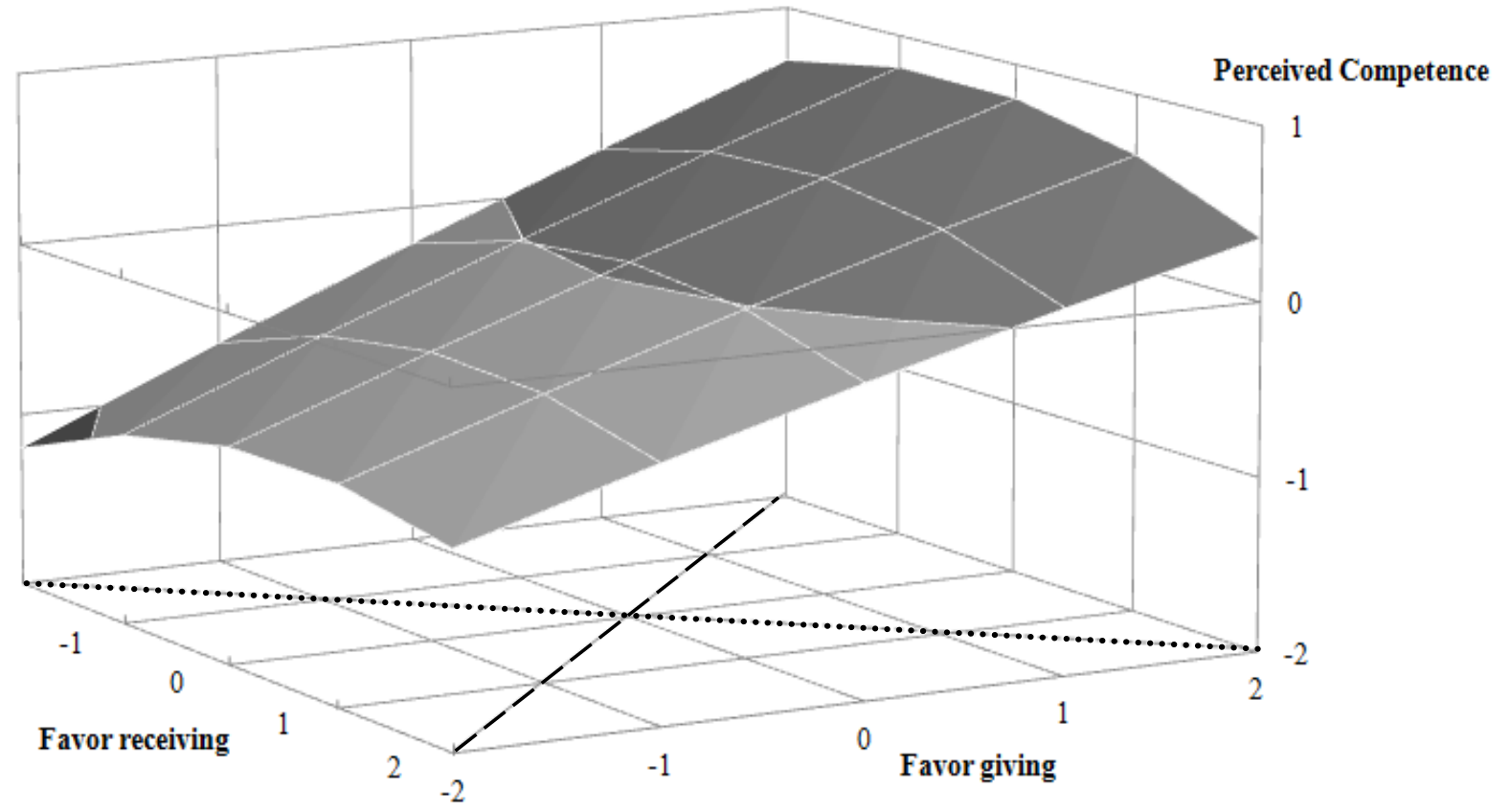

Figure 5. Effects of generous, stingy, and matched favor giving on perceived competence, Study 2. Following Zhang and colleagues (2012), for better visual examination, we changed the orientation in the response surface from what Edwards and Parry (1993) used. The long-dashed line represents the generous/stingy line, along which favor giving becomes more generous from the front corner to the rear corner; the round-dotted line represents the matched line, along which the frequency of matched favor giving increases from the left corner to the right corner. 
$-2$

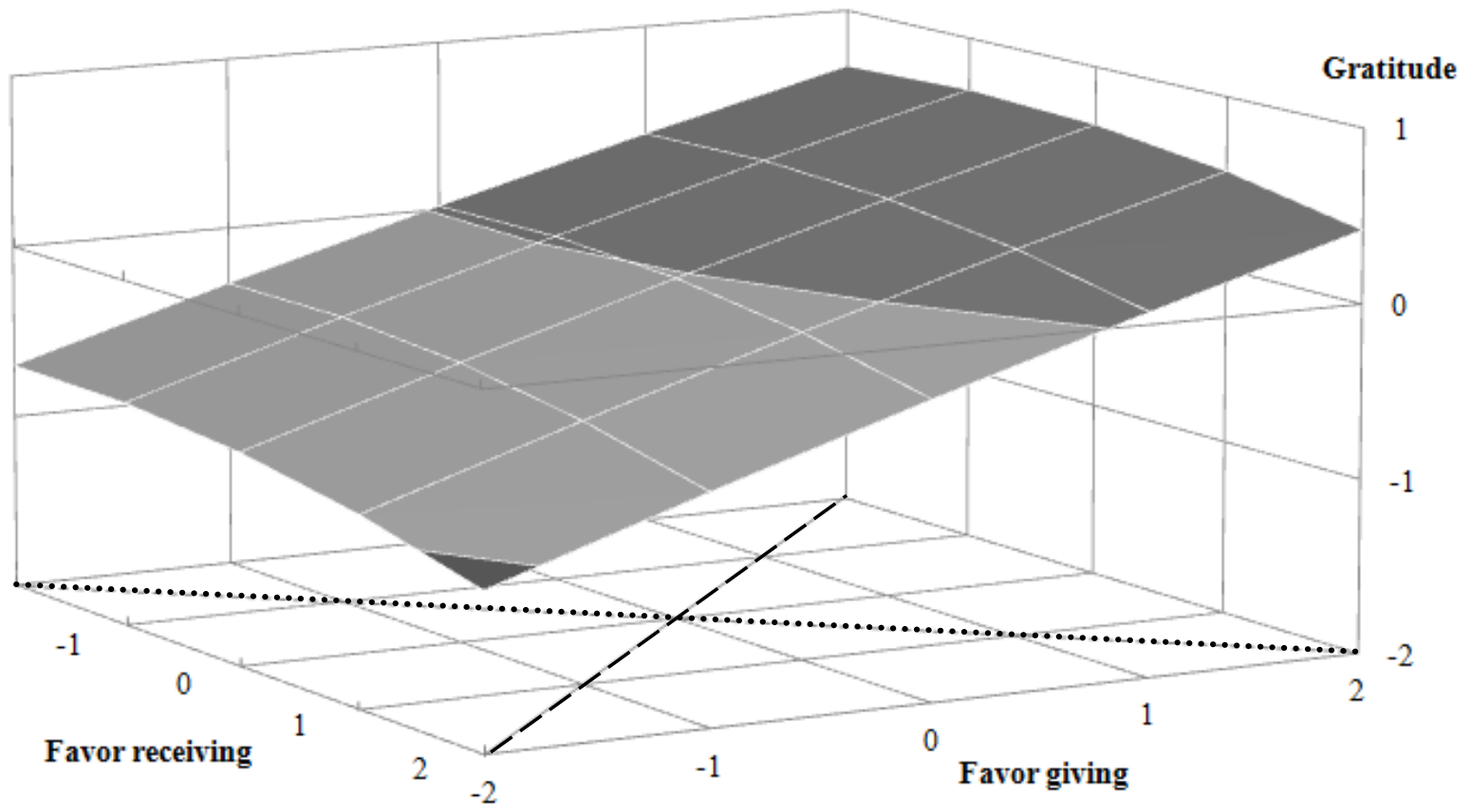

Figure 6. Effects of generous, stingy, and matched favor giving on gratitude, Study 2. Following Zhang and colleagues (2012), for better visual examination, we changed the orientation in the response surface from what Edwards and Parry (1993) used. The long-dashed line represents the generous/stingy line, along which favor giving becomes more generous from the front corner to the rear corner; the round-dotted line represents the matched line, along which the frequency of matched favor giving increases from the left corner to the right corner. 


\section{Appendix}

\section{Flow of Procedure and Instructions of Study 1}

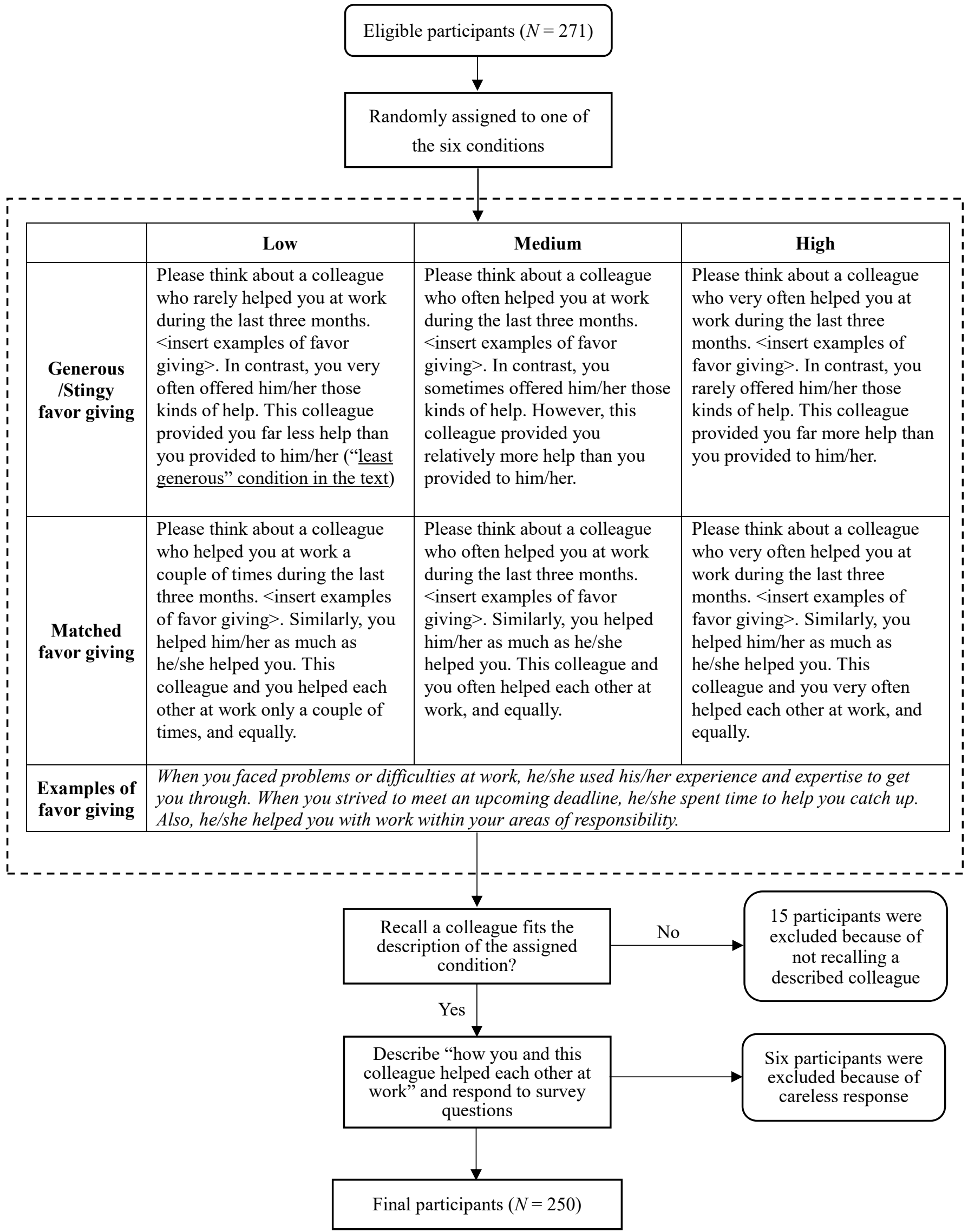

